\title{
One-Dimensional Impenetrable Anyons in Thermal Equilibrium. III. Large distance asymptotics of the space correlations.
}

\author{
Ovidiu I. Pâţu, ${ }^{1,2}$ Vladimir E. Korepin, ${ }^{1}$ and Dmitri V. Averin ${ }^{3}$ \\ ${ }^{1}$ C.N. Yang Institute for Theoretical Physics, State University of New York at Stony Brook, Stony Brook, NY 11794-3840, USA \\ ${ }^{2}$ Institute for Space Sciences, Bucharest-Măgurele, R 077125, Romania \\ ${ }^{3}$ Department of Physics and Astronomy, State University of New York at Stony Brook, Stony Brook, NY 11794-3800, USA *
}

\begin{abstract}
Using the determinant representation for the field-field correlation functions of impenetrable anyons at finite temperature obtained in [1], we derive a system of nonlinear partial differential equations completely characterizing the correlators. The system is the same as the one for impenetrable bosons but with different initial conditions. The large-distance asymptotic behavior of the correlation functions is obtained from the analysis of the Riemann-Hilbert problem associated with the system of differential equations. We calculate both the exponential and pre-exponential factors in the asymptotics of the field-field correlators. The asymptotics derived in this way agree with those of the free fermions and impenetrable bosons in the appropriate limits, $\kappa \rightarrow 1$ and $\kappa \rightarrow 0$, of the statistics parameter $\kappa$, and coincide with the predictions of the conformal field theory at low temperatures.
\end{abstract}

PACS numbers: 02.30Ik, 05.30.Pr

\section{INTRODUCTION AND STATEMENT OF RESULTS}

This is the third paper in the series investigating the correlation functions of one-dimensional impenetrable anyons at finite temperatures. In the previous two papers [1, 2], we have obtained the anyonic generalization of Lenard's formula and determinant representations for the time-, space-, and temperature-dependent correlators of the anyons. Here, starting from the obtained determinant representation, we derive the explicit expressions for the large-distance asymptotics of the anyonic space correlators at finite temperatures. These expressions demonstrate the crossover between the fermionic and bosonic behavior of the correlators when the appropriately-defined statistics parameter $\kappa \in[0,1]$ changes between the corresponding limits of bosons, $\kappa=0$, and fermions, $\kappa=1$. The approach we use to compute the asymptotics of the correlation functions of impenetrable anyons follows the one developed for impenetrable bosons [3, 4, 5]. The main technical point of this approach is the large-distance asymptotic analysis of the Riemann-Hilbert problem associated with the system of differential equations which can be derived from the determinant representation.

The model of impenetrable one-dimensional (1D) anyons we consider is the limit of infinite interaction strength of the Lieb-Liniger gas of anyons with delta-functional repulsion [6, 7, 8]. The fact that impenetrable particles can not be directly exchanged in 1D systems implies that introduction of the exchange statistics for such particles requires an additional convention on the choice of the sign of the statistical phase associated with exchange of each pair of particles in the system wavefunctions [9, 10]. The absence of direct exchanges also implies that local thermodynamic properties of $N$ impenetrable 1D anyons are independent of statistics in the thermodynamic limit $N \rightarrow \infty$, and coincide with those of the free fermions - see, e.g. [11]. In the case of quasiperiodic boundary conditions (particles moving on a circle), thermodynamic properties are statistics-dependent at the level of corrections to the leading large- $N$ asymptotic terms [12, 13, 14, 15]. Although the non-local characteristics like single-particle momentum distribution (i.e., Fourier transform of the single-particle density matrix) depend strongly on the statistics parameter $\kappa$ [15, 16], they may not be measurable in practice [17]. For transparent anyons, i.e. finite particle-particle repulsion, local thermodynamic properties do depend on $\kappa$ through modification of the effective coupling constant [10, 13, 18, 19], but the model of anyons without a hard core is not well-defined because of the essential singularity of the system wavefunction at coincident particle coordinates. Qualitatively, these properties of the one-dimensional anyons are preserved in other related anyonic models with, e.g., motion on the lattice 20, 21, 22, 23] or different interactions [24, 25].

This work presents the exact calculation of the large-distance asymptotic of the field-field correlator of impenetrable

*Electronic addresses: ipatu@grad.physics.sunysb.edu; korepin@max2.physics.sunysb.edu ; dmitri.averin@stonybrook.edu 
anyons. To state its main result, we need some notations and definitions. First, we introduce the function

$$
\nu(\lambda, \beta)=\frac{1}{2 \pi i} \ln \left(\frac{e^{\lambda^{2}-\beta}+1}{e^{\lambda^{2}-\beta}-e^{i \pi \kappa}}\right)
$$

where the branch of the logarithm is specified by the requirements that no branch cut intersects the real axis, and

$$
\lim _{\lambda \rightarrow \infty} \ln \left(\frac{e^{\lambda^{2}-\beta}+1}{e^{\lambda^{2}-\beta}-e^{i \pi \kappa}}\right) \rightarrow 0
$$

We then define two constants $C(\beta, \kappa)$ and $c(\beta, \kappa)$ as

$$
C(\beta, \kappa)=2 i \int_{-\infty}^{+\infty} \nu(\lambda, \beta) d \lambda=\frac{1}{\pi} \int_{-\infty}^{+\infty} \ln \left(\frac{e^{\lambda^{2}-\beta}+1}{e^{\lambda^{2}-\beta}-e^{i \pi \kappa}}\right) d \lambda
$$

and

$$
c(\beta, \kappa)=\int_{-\infty}^{+\infty} \frac{\partial_{\lambda} \nu(\lambda, \beta) \nu(\mu, \beta)-\nu(\lambda, \beta) \partial_{\mu} \nu(\mu, \beta)}{2(\lambda-\mu)} d \lambda d \mu
$$

In terms of these quantities, our main result for the two leading terms in the large-distance asymptotic of the field-field correlator of impenetrable anyons is:

$$
\left\langle\Psi^{\dagger}\left(x_{1}\right) \Psi\left(x_{2}\right)\right\rangle_{T}=e^{-x_{12} \sqrt{T} C(h / T, \kappa) / 2} e^{c(h / T, \kappa)}\left(c_{0} e^{i x_{12} \sqrt{T} \lambda_{0}}+c_{-1} e^{i x_{12} \sqrt{T} \lambda_{-1}}\right)
$$

Here $T$ is temperature, $h$ - chemical potential, $x_{12} \equiv\left(x_{1}-x_{2}\right) \rightarrow+\infty$, and the constants $\lambda_{j}$ with $j=0,-1$ are complex numbers which depend on $\beta \equiv h / T$ and statistics $\kappa$ as

$$
\lambda_{j}=\left(\beta+\sqrt{\beta^{2}+\pi^{2}[\kappa+2 j]^{2}}\right)^{1 / 2} / \sqrt{2}+i\left(-\beta+\sqrt{\beta^{2}+\pi^{2}[\kappa+2 j]^{2}}\right)^{1 / 2} / \sqrt{2} .
$$

Two other constants $c_{j}$ which give the amplitudes of the asymptotic terms in (5) are given by

$$
c_{j}=i \frac{e^{i \pi \kappa} \sqrt{T}}{2} \frac{\alpha^{-2}\left(\lambda_{j}\right)}{\lambda_{j}},
$$

where the function $\alpha(\lambda)$ is defined by Eq. (53). The second term in the asymptotic expansion (5) is always smaller than the first one, but becomes relevant when the statistics parameter approaches the fermionic value $\kappa=1$. The precise conditions on $\kappa$ under which this term does not exceed the accuracy of our calculation are stated in Section $\mathrm{VA}$, see Eqs. (72) and (73). More generally, all results of this work are obtained, strictly speaking, only for $\kappa \in(0,1]$. However, as shown in Section VIA in the limit $\kappa \rightarrow 0$ our results reproduce completely the exponential behavior of the asymptotics for impenetrable bosons $(\kappa=0)$ [5, 41], and the pre-exponential factors in the case of negative chemical potential $h$.

An important role in obtaining the asymptotics (5) is played by a set of auxiliary potentials $B_{+-}$and $B_{++}$ introduced below in Section II which are related to the integral operators in the representation of the correlation functions. The integral operator which enters in the Fredholm determinant representation for the correlator is of a special kind called "integrable integral operator". Integral operators of this type play an important role in the study of correlation functions of integrable models and random matrices [3, 4, 29, 30, 31, 32]. The special form of the kernels of such operators allowed us to obtain a system of partial differential equations for the auxiliary potentials, and for the logarithm of the Fredholm determinant, which characterize completely the correlators at any distance. The system of equations is the same as the one obtained for impenetrable bosons [3, 5, 33] but with different initial conditions. The short-distance and low-density behavior of the field-field correlator can be extracted from the initial conditions. At zero temperature, the nonlinear differential equation for the logarithm of the determinant becomes the Painlevé $\mathrm{V}$ differential equation obtained by Jimbo, Miwa, Môri and Sato for impenetrable bosons [30], but again, with different initial conditions. For finite system, the Painlevé VI differential equation characterizing the field-field correlator was derived by Santachiara and Calabrese in [34]. The short-distance behavior of the 2-point correlation function obtained in [34] agrees in the thermodynamic limit with our results at zero temperature. The large-distance behavior of the correlation functions is extracted from the solution of a matrix Riemann-Hilbert problem associated with the system of 
differential equations for the auxiliary potentials. The technique used is similar to the one developed for impenetrable bosons [3, 5, 33]. For the computation of the pre-exponential factors in the asymptotics of the correlators we have adopted the method employed by Kitanine, Kozlowski, Maillet, Slavnov and Terras in their study of the generalized sine-kernel [36]. This method was first used by Cheianov and Zvonarev in the computation of the asymptotic behavior of correlation functions of impenetrable electrons [26, 27]. Some of the results of this work were presented in [28].

The plan of the paper is as follows. In Section II, we briefly review the determinant representation for correlators obtained in our previous papers [1, 2], and introduce the auxiliary potentials which play a central role in the subsequent analysis of the correlation functions. In Section [II, we derive the system of partial nonlinear differential equations characterizing the auxiliary potentials and the correlators. Section IV introduces the matrix Riemann-Hilbert problem associated with the obtained system of equations. The large-distance analysis of this problem is performed in Section V] We conclude in Section VI by comparing our main result with the known limiting cases: the low-temperature limit characterized by the conformal behavior of the correlators, and bosonic and fermionic limits of the statistics parameter. Technical details of the calculations are presented in the appendices.

\section{DETERMINANT REPRESENTATION AND AUXILIARY POTENTIALS}

The model of one-dimensional anyons we consider in this work is characterized by the hamiltonian $H$ given in the second quantized form as

$$
H=\int d x\left(\left[\partial_{x} \Psi^{\dagger}(x)\right]\left[\partial_{x} \Psi(x)\right]+c \Psi^{\dagger}(x) \Psi^{\dagger}(x) \Psi(x) \Psi(x)-h \Psi^{\dagger}(x) \Psi(x)\right) .
$$

Here $c$ is the coupling constant which is assumed to be large, $c \rightarrow+\infty$, so that the anyons are impenetrable. The fields in the Hamiltonian (8) obey the anyonic commutation relations

$$
\begin{gathered}
\Psi\left(x_{1}\right) \Psi^{\dagger}\left(x_{2}\right)=e^{-i \pi \kappa \epsilon\left(x_{1}-x_{2}\right)} \Psi^{\dagger}\left(x_{2}\right) \Psi\left(x_{1}\right)+\delta\left(x_{1}-x_{2}\right), \\
\Psi^{\dagger}\left(x_{1}\right) \Psi^{\dagger}\left(x_{2}\right)=e^{i \pi \kappa \epsilon\left(x_{1}-x_{2}\right)} \Psi^{\dagger}\left(x_{2}\right) \Psi^{\dagger}\left(x_{1}\right),
\end{gathered}
$$

where $\epsilon(x)=x /|x|, \epsilon(0)=0$. The commutation relations become bosonic for $\kappa=0$, and fermionic for $\kappa=1$. In this work, we are interested in the large-distance behavior of the field-field correlator of the impenetrable anyons (8). The static, i.e. equal-time, correlator at finite temperatures is defined by the standard relation:

$$
\left\langle\Psi^{\dagger}\left(x_{1}\right) \Psi\left(x_{2}\right)\right\rangle_{T} \equiv \frac{\operatorname{Tr}\left(e^{-H / T} \Psi^{\dagger}\left(x_{1}\right) \Psi\left(x_{2}\right)\right)}{\operatorname{Tr} e^{-H / T}} .
$$

In the previous paper [2] of the series, we have obtained the following expressions for this correlator

$$
\left\langle\Psi^{\dagger}\left(x_{1}\right) \Psi\left(x_{2}\right)\right\rangle_{T}=\left.\frac{1}{2 \pi} \operatorname{Tr}\left[\left(1-\gamma \hat{K}_{T}\right)^{-1} \hat{A}_{T}^{+}\right] \operatorname{det}\left(1-\gamma \hat{K}_{T}\right)\right|_{\gamma=\left(1+e^{+i \pi \kappa}\right) / \pi}, \quad x_{1}>x_{2},
$$

and

$$
\left\langle\Psi^{\dagger}\left(x_{1}\right) \Psi\left(x_{2}\right)\right\rangle_{T}=\left.\frac{1}{2 \pi} \operatorname{Tr}\left[\left(1-\gamma \hat{K}_{T}\right)^{-1} \hat{A}_{T}^{-}\right] \operatorname{det}\left(1-\gamma \hat{K}_{T}\right)\right|_{\gamma=\left(1+e^{-i \pi \kappa}\right) / \pi}, \quad x_{1}<x_{2} .
$$

In these expressions, $\hat{K}_{T}$ and $\hat{A}_{T}^{ \pm}$are the integral operators acting on the entire real axis with the kernels

$$
K_{T}(\lambda, \mu)=\sqrt{\vartheta(\lambda)} \frac{\sin x_{12}(\lambda-\mu)}{\lambda-\mu} \sqrt{\vartheta(\mu)}, \quad A_{T}^{ \pm}(\lambda, \mu)=\sqrt{\vartheta(\lambda)} e^{\mp i x_{12}(\lambda+\mu)} \sqrt{\vartheta(\mu)}
$$

where

$$
\vartheta(\lambda) \equiv \vartheta(\lambda, T, h)=\frac{1}{1+e^{\left(\lambda^{2}-h\right) / T}},
$$

is the Fermi distribution function, and $\operatorname{Tr}[f(x, y)] \equiv \int f(x, x) d x$. Equations (10) and (11) remain valid at zero temperature, but in this case, the integral operators act on the interval $[-q, q]$ with $q=\sqrt{h}$, and have kernels

$$
K(\lambda, \mu)=\frac{\sin x_{12}(\lambda-\mu)}{\lambda-\mu}, \quad A^{ \pm}(\lambda, \mu)=e^{\mp i x_{12}(\lambda+\mu)} .
$$


Equations (10) and (11) show that the anyonic corrrelator $\left\langle\Psi^{\dagger}\left(x_{1}\right) \Psi\left(x_{2}\right)\right\rangle_{T}$ depends on the sign of $x_{1}-x_{2}$. However, since the values (10) and (11) for positive and negative $x_{1}-x_{2}$ are related directly via complex conjugation, one can focus only on one range, e.g., the correlator (10). This correlator depends on four variables: the coordinate difference $x_{1}-x_{2}>0$, temperature $T$, chemical potential $h$, and statistics parameter $\kappa$. As we will see bellow, introducing the variables that are rescaled by temperature: the distance $x$ and the chemical potential $\beta$ defined as

$$
x=\frac{1}{2}\left(x_{1}-x_{2}\right) \sqrt{T}, \quad \beta=\frac{h}{T},
$$

and similarly changing the spectral parameter, $\lambda \rightarrow \lambda \sqrt{T}$, one makes explicit dependence on temperature very simple:

$$
\left\langle\Psi^{\dagger}\left(x_{1}\right) \Psi\left(x_{2}\right)\right\rangle_{T}=\left.\frac{\sqrt{T}}{2 \pi \gamma} g(x, \beta, \gamma)\right|_{\gamma=\left(1+e^{i \pi \kappa}\right) / \pi} .
$$

The function $g(x, \beta, \gamma)$ here will be defined in the next section.

\section{A. Auxiliary Potentials}

The Fredholm integral operator $\hat{K}_{T}$ appearing in the expressions (10) and (11) for the field correlators belongs to a special class of "integrable" operators [3, 4, 32]. This means that in terms of the "plane waves" introduced as

$$
e_{ \pm}(\lambda)=\sqrt{\vartheta(\lambda)} e^{ \pm i \lambda x}
$$

the kernel of $\hat{K}_{T}$ can be written in the following form which generalizes the simple factorizable kernels:

$$
K_{T}(\lambda, \mu)=\frac{e_{+}(\lambda) e_{-}(\mu)-e_{-}(\lambda) e_{+}(\mu)}{2 i(\lambda-\mu)} .
$$

This is a particular case of the more general situation studied in [4, 32] (see also Chap XIV of [3]). An important feature of this class of integrable operators is the fact that the kernel $R_{T}(\lambda, \mu)$ of the resolvent operator $\hat{R}_{T}$, defined by the relation

$$
\hat{R}_{T}=\left(1-\gamma \hat{K}_{T}\right)^{-1} \hat{K}_{T} \text {, i.e., }\left(1-\gamma \hat{K}_{T}\right)\left(1+\gamma \hat{R}_{T}\right)=1 \text {, }
$$

has the same form [3, 4]. Indeed, Eq. (16) means that the resolvent kernel $R_{T}(\lambda, \mu)$ solves the integral equation

$$
R_{T}(\lambda, \mu)-\gamma \int_{-\infty}^{+\infty} K_{T}(\lambda, \nu) R_{T}(\nu, \mu) d \nu=K_{T}(\lambda, \mu) .
$$

Introducing then the functions $f_{ \pm}(\lambda)$ which are the solutions of the similar integral equations

$$
f_{ \pm}(\lambda)-\gamma \int_{-\infty}^{+\infty} K_{T}(\lambda, \mu) f_{ \pm}(\mu) d \mu=e_{ \pm}(\lambda)
$$

one can show (for a proof, see Chap XIV of [3] ) that the resolvent kernel can be written in the same form (15]) as $K_{T}$ :

$$
R_{T}(\lambda, \mu)=\frac{f_{+}(\lambda) f_{-}(\mu)-f_{-}(\lambda) f_{+}(\mu)}{2 i(\lambda-\mu)} .
$$

An important role in our asymptotic analysis of the correlator (10) is played by the auxiliary potentials $B_{l m}$ which are defined by

$$
B_{l m}(x, \beta, \kappa) \equiv \gamma \int_{-\infty}^{+\infty} e_{l}(\lambda) f_{m}(\lambda) d \lambda, \quad l= \pm, m= \pm
$$

where $\gamma=\left(1+e^{i \pi \kappa}\right) / \pi$. A new feature of the auxiliary potentials $B_{l m}$ in the case of anyons in comparison to bosons, when $\gamma=2 / \pi$, is that they are now complex. The potentials, however, still satisfy the relations $B_{+-}(x, \beta, \kappa)=$ 
$B_{-+}(x, \beta, \kappa)$ and $B_{++}(x, \beta, \kappa)=B_{--}(x, \beta, \kappa)$ as in the bosonic case. Indeed, as one can see from the definitions (19) and (17)

$$
\begin{aligned}
B_{+-} & =\gamma \int_{-\infty}^{+\infty} e_{+}(\lambda) f_{-}(\lambda) d \lambda=\gamma \int_{-\infty}^{+\infty} e_{+}(\lambda) \int_{-\infty}^{+\infty}\left(1-\gamma \hat{K}_{T}\right)^{-1}(\lambda, \mu) e_{-}(\mu) d \mu d \lambda \\
& =\gamma \int_{-\infty}^{+\infty} e_{-}(\lambda) f_{+}(\lambda) d \lambda \equiv B_{-+},
\end{aligned}
$$

where in the last line we have used the fact that the kernel $K_{T}$ is symmetric, $K_{T}(\lambda, \mu)=K_{T}(\mu, \lambda)$. In order to prove the second assertion we start with the integral equation (17) for $f_{+}(-\lambda)$

$$
f_{+}(-\lambda)-\gamma \int_{-\infty}^{+\infty} K_{T}(-\lambda, \mu) f_{+}(\mu) d \mu=\sqrt{\vartheta(\lambda)} e^{-i \lambda x}
$$

which can be rewritten as

$$
f_{+}(-\lambda)-\gamma \int_{-\infty}^{+\infty} K_{T}(-\lambda,-\mu) f_{+}(-\mu) d \mu=\sqrt{\vartheta(\lambda)} e^{-i \lambda x}
$$

Using the relation $K_{T}(-\lambda,-\mu)=K_{T}(\lambda, \mu)$, we see then that $f_{+}(-\lambda)=f_{-}(\lambda)$. This gives

$$
\begin{aligned}
B_{++} & =\gamma \int_{-\infty}^{+\infty} e_{+}(\lambda) f_{+}(\lambda) d \lambda=\gamma \int_{-\infty}^{+\infty} e_{+}(-\lambda) f_{+}(-\lambda) d \lambda \\
& =\gamma \int_{-\infty}^{+\infty} e_{-}(\lambda) f_{-}(\lambda) d \lambda=B_{--}
\end{aligned}
$$

Finally, the definition of the kernel $A_{T}^{+}$shows directly that (10) can be rewritten as

$$
\left\langle\Psi^{\dagger}\left(x_{1}\right) \Psi\left(x_{2}\right)\right\rangle_{T}=\left.\frac{\sqrt{T}}{2 \pi} \operatorname{det}\left(1-\gamma \hat{K}_{T}\right)\right|_{\gamma=\left(1+e^{+i \pi \kappa}\right) / \pi} \int_{-\infty}^{+\infty} f_{-}(\lambda) e_{-}(\lambda) d \lambda,
$$

in terms of the function $f_{-}(\lambda)$ (17). This shows that the function $g(x, \beta, \gamma)$ introduced in Eq. (13) is given by

$$
g(x, \beta, \gamma)=\left.B_{++}(x, \beta, \gamma) \operatorname{det}\left(1-\gamma \hat{K}_{T}\right)\right|_{\gamma=\left(1+e^{+i \pi \kappa}\right) / \pi}
$$

\section{DIFFERENTIAL EQUATIONS FOR STATIC CORRELATORS}

In general, it is difficult to obtain directly differential equations for the entire correlator (10). The strategy we pursue is to obtain first nonlinear partial differential equations for the potentials $B_{++}, B_{+-}$, and then show that the function $\sigma(x, \beta, \gamma)=\ln \operatorname{det}\left(1-\gamma \hat{K}_{T}\right)$ can be expressed in terms of $B_{++}$and $B_{+-}$. We start by look for the two operators $\mathrm{L}$ and $\mathrm{M}$ depending on $B_{++}$and $B_{+-}$such that we have

$$
\begin{aligned}
\partial_{x} F(\lambda) & =\mathrm{L} F(\lambda), \\
\left(2 \lambda \partial_{\beta}+\partial_{\lambda}\right) F(\lambda) & =\mathrm{M} F(\lambda),
\end{aligned}
$$

where $F(\lambda)$ is the two-component vector function

$$
F(\lambda)=\left(\begin{array}{c}
f_{+}(\lambda) \\
f_{-}(\lambda)
\end{array}\right)
$$

The compatibility condition for the system (21)

$$
\left(2 \lambda \partial_{\beta}+\partial_{\lambda}\right) \mathrm{L}-\partial_{x} \mathrm{M}+[\mathrm{L}, \mathrm{M}]=0,
$$

gives then a system of partial differential equations in $x$ and $\beta$. It should be noted that since the Fredholm determinant in Eq. (10) differs from the one appearing in the similar representation for impenetrable bosons only in the value of $\gamma$, the calculations follow closely those for impenetrable bosons [3, 33]. The main difference is that $\gamma$ for anyons is complex, making the auxiliary potentials $B_{++}, B_{+-}$complex and not real as for the bosons. 


\section{A. The L operator}

We start from the integral equations for the functions $f_{ \pm}(\lambda)$

$$
f_{ \pm}(\lambda)-\gamma \int_{-\infty}^{+\infty} K_{T}(\lambda, \mu) f_{ \pm}(\mu) d \mu=e_{ \pm}(\lambda), \quad \gamma=\left(1+e^{i \pi \kappa}\right) / \pi
$$

Differentiation with respect to $x$ gives

$$
\left[\left(1-\gamma \hat{K}_{T}\right) \partial_{x} f_{ \pm}\right](\lambda)-\gamma\left[\partial_{x} \hat{K}_{T} f_{ \pm}\right](\lambda)= \pm i \lambda e_{ \pm}(\lambda) .
$$

Acting on both sides with $\left(1-\gamma \hat{K}_{T}\right)^{-1}$ and using the relations $\left(1-\gamma \hat{K}_{T}\right)^{-1}=\left(1+\gamma \hat{R}_{T}\right),\left[\left(1-\gamma \hat{K}_{T}\right)^{-1} e_{ \pm}\right](\lambda)=f_{ \pm}(\lambda)$, $\partial_{x} K_{T}(\lambda, \mu)=\left(e_{+}(\lambda) e_{-}(\mu)+e_{-}(\lambda) e_{+}(\mu)\right) / 2$, and the special factorization for the resolvent kernel (18) we obtain

$$
\begin{aligned}
& \partial_{x} f_{+}(\lambda)=i \lambda f_{+}(\lambda)+B_{++} f_{-}(\lambda), \\
& \partial_{x} f_{-}(\lambda)=-i \lambda f_{+}(\lambda)+B_{--} f_{-}(\lambda) .
\end{aligned}
$$

These equations mean that the $L$ operator has the form

$$
\mathbf{L}=i \lambda \sigma_{3}+B_{++} \sigma_{1}
$$

where we have used the fact that $B_{++}=B_{--}$, and $\sigma_{i}$ are the Pauli matrices

$$
\sigma_{1}=\left(\begin{array}{ll}
0 & 1 \\
1 & 0
\end{array}\right), \quad \sigma_{2}=\left(\begin{array}{rr}
0 & -i \\
i & 0
\end{array}\right), \quad \sigma_{3}=\left(\begin{array}{rr}
1 & 0 \\
0 & -1
\end{array}\right) .
$$

\section{B. The M operator}

The derivation of the $\mathrm{M}$ operator is more complicated. In this case, we have to rely heavily on the following property of the Fermi distribution function $\vartheta(\lambda)$ :

$$
\left(2 \lambda \partial_{\beta}+\partial_{\lambda}\right) \vartheta(\lambda)=0 .
$$

This property is essential, since in the derivation of the $\mathrm{M}$ operator, one encounters terms which contain the resolvent $R_{T}(\lambda, \mu)$ but still can not be reduced to the form $(\lambda-\mu) R_{T}(\lambda, \mu)$ (as in the previous section) which produces "disentangled" terms (i.e., products of the one-dimensional projectors). This is also why the differential operator associated with $\mathrm{M}$ is $\left(2 \lambda \partial_{\beta}+\partial_{\lambda}\right)$ and not simply $\partial_{\beta}$ as one could have expected.

The computations are more involved but similar with the ones for impenetrable bosons and we refer the reader to Chap. XIV of [3] for the derivation. The final result is

$$
\begin{aligned}
& \left(2 \lambda \partial_{\beta}+\partial_{\lambda}\right) f_{+}(\lambda)=i x f_{+}(\lambda)-i f_{+}(\lambda) \partial_{\beta} B_{-+}+i f_{-}(\lambda) \partial_{\beta} B_{++} \\
& \left(2 \lambda \partial_{\beta}+\partial_{\lambda}\right) f_{-}(\lambda)=-i x f_{+}(\lambda)-i f_{+}(\lambda) \partial_{\beta} B_{--}+i f_{-}(\lambda) \partial_{\beta} B_{+-}
\end{aligned}
$$

Taking into account that $B_{-+}=B_{+-}, B_{++}=B_{--}$this finally gives the $\mathrm{M}$ operator as

$$
\mathrm{M}=i x \sigma_{3}-i \partial_{\beta} B_{+-} \sigma_{3}-\partial_{\beta} B_{++} \sigma_{2} .
$$

\section{Differential equations for the potentials}

The results obtained in the previous sections allow us to state the following theorem:

Theorem III.1. For all $\gamma=\left(1+e^{i \pi \kappa}\right) / \pi$ with $\kappa \in[0,1)$, the potentials $B_{+-}(x, \beta, \gamma), B_{++}(x, \beta, \gamma)$ satisfy the following system of partial differential equations

$$
\begin{aligned}
& \partial_{\beta} B_{+-}=x+\frac{1}{2} \frac{\partial_{x} \partial_{\beta} B_{++}}{B_{++}}, \\
& \partial_{x} B_{+-}=B_{++}^{2},
\end{aligned}
$$


with the initial conditions (at fixed $\beta$ )

$$
B_{++}(x, \beta, \gamma)=B_{+-}(x, \beta, \gamma)=\gamma d(\beta)+[\gamma d(\beta)]^{2} x+O\left(x^{2}\right), \quad x \rightarrow 0,
$$

where $d(\beta)=\int_{-\infty}^{+\infty} \vartheta(\lambda) d \lambda$ and

$$
B_{++}(x, \beta, \gamma)=B_{+-}(x, \beta, \gamma)=0, \beta \rightarrow-\infty
$$

The potential $B_{++}(x, \beta, \gamma)$ satisfies the nonlinear equation

$$
\partial_{\beta} B_{++}^{2}=1+\frac{1}{2} \frac{\partial}{\partial_{x}}\left(\frac{\partial_{x} \partial_{\beta} B_{++}}{B_{++}}\right),
$$

with the same initial conditions.

Proof. In the previous sections we have shown that the two-component vector function $F(\lambda)$ satisfies the following differential equations

$$
\begin{aligned}
\partial_{x} F(\lambda) & =\mathrm{L} F(\lambda), \\
\left(2 \lambda \partial_{\beta}+\partial_{\lambda}\right) F(\lambda) & =\mathrm{M} F(\lambda) .
\end{aligned}
$$

where L,M are given by (23) and (25). The compatibility condition for these equation is

$$
\left[\partial_{x}-\mathrm{L},\left(2 \lambda \partial_{\beta}+\partial_{\lambda}\right)-\mathrm{M}\right]=\left(2 \lambda \partial_{\beta}+\partial_{\lambda}\right) \mathrm{L}-\partial_{x} \mathrm{M}+[\mathrm{L}, \mathrm{M}]=0 .
$$

Differentiating Eqs. (23) and (25), we get

$$
\begin{gathered}
\left(2 \lambda \partial_{\beta}+\partial_{\lambda}\right) \mathrm{L}=2 \lambda\left(\partial_{\beta} B_{++}\right) \sigma_{1}+i \sigma_{3}, \\
\partial_{x} \mathrm{M}=i \sigma_{3}-i\left(\partial_{x} \partial_{\beta} B_{+-}\right) \sigma_{3}-\left(\partial_{x} \partial_{\beta} B_{++}\right) \sigma_{2} .
\end{gathered}
$$

Using the standard relation for the Pauli matrices

$$
\left[\sigma_{i}, \sigma_{j}\right]=2 i \epsilon_{i j k} \sigma_{k}
$$

we also have

$$
[\mathrm{L}, \mathrm{M}]=-2 \lambda\left(\partial_{\beta} B_{++}\right) \sigma_{1}+\left[2 x B_{++}-2 B_{++}\left(\partial_{\beta} B_{+-}\right)\right] \sigma_{2}-2 i B_{++}\left(\partial_{\beta} B_{++}\right) \sigma_{3} .
$$

Plugging these results into Eq. (31) we obtain a matrix with only two distinct elements. The condition that this matrix vanishes identically gives the following relations

$$
2 B_{++}\left(\partial_{\beta} B_{+-}\right)-2 x B_{++}+\partial_{x} \partial_{\beta} B_{++}=0
$$

and

$$
\partial_{x} \partial_{\beta} B_{+-}-\partial_{\beta} B_{++}^{2}=0
$$

The first relation is just Eq. (26), while the second is equivalent to Eq. (27) due to (29). The equation (30) is obtained by differentiating (26) with respect to $x$, 27) with respect to $\beta$, and then equating the right-hand sides of the resulting relations. The asymptotic behavior at short distances (28) and at low density (29), which give the initial conditions, are obtained in Appendices (A) and (B).

\section{Differential equations for $\sigma$}

Theorem III.2. For any $\gamma=\left(1+e^{i \pi \kappa}\right) / \pi$ with $\kappa \in[0,1)$, the partial derivatives of $\sigma(x, \beta, \gamma) \equiv \ln \operatorname{det}\left(1-\gamma \hat{K}_{T}\right)$ with respect to $x$ and $\beta$ are given by

$$
\begin{aligned}
& \partial_{x} \sigma=-B_{+-}, \quad \partial_{x}^{2} \sigma=-B_{++}^{2} \\
& \partial_{\beta} \sigma=-x \partial_{\beta} B_{+-}+\frac{1}{2}\left(\partial_{\beta} B_{+-}\right)^{2}-\frac{1}{2}\left(\partial_{\beta} B_{++}\right)^{2} .
\end{aligned}
$$


Furthermore, for all $\gamma$ s, the function $\sigma(x, \beta, \gamma)$ satisfies the following nonlinear partial differential equation

$$
\left(\partial_{\beta} \partial_{x}^{2} \sigma\right)^{2}+4\left(\partial_{x}^{2} \sigma\right)\left[2 x \partial_{\beta} \partial_{x} \sigma+\left(\partial_{\beta} \partial_{x} \sigma\right)^{2}-2 \partial_{\beta} \sigma\right]=0
$$

with the initial conditions

$$
\sigma=-\gamma d(\beta) x-[\gamma d(\beta)]^{2} \frac{x^{2}}{2}+O\left(x^{3}\right), \quad x \rightarrow 0 ; \quad \sigma=0, \quad \beta \rightarrow-\infty,
$$

where, as before, $d(\beta)=\int_{-\infty}^{+\infty} \vartheta(\lambda) d \lambda$.

Proof. We start with the simpler case of derivative with respect to $x$. Making use of the following standard representation for the Fredholm determinant of an arbitrary integral operator

$$
\operatorname{det}\left(1-\gamma \hat{K}_{T}\right)=\exp \left(-\sum_{n=1}^{\infty} \frac{\gamma^{n}}{n} \operatorname{Tr} K_{T}^{n}\right),
$$

where $\operatorname{Tr} K_{T}=\int K_{T}(\lambda, \lambda), \quad \operatorname{Tr} K_{T}^{2}=\int K_{T}(\lambda, \mu) K_{T}(\mu, \lambda) d \lambda d \mu$, and so on, we obtain

$$
\partial_{x} \sigma=-\gamma \operatorname{Tr}\left[\left(1-\gamma \hat{K}_{T}\right)^{-1} \partial_{x} \hat{K}_{T}\right] .
$$

From the relations $\left[\left(1-\gamma \hat{K}_{T}\right)^{-1} e_{ \pm}\right](\lambda)=f_{ \pm}(\lambda)$ and $\partial_{x} K_{T}(\lambda, \mu)=\left(e_{+}(\lambda) e_{-}(\mu)+e_{-}(\lambda) e_{+}(\mu)\right) / 2$ that were already used before, we have

$$
\begin{aligned}
-\gamma \operatorname{Tr}\left[\left(1-\gamma \hat{K}_{T}\right)^{-1} \partial_{x} \hat{K}_{T}\right] & =-\frac{\gamma}{2} \int_{-\infty}^{+\infty} f_{+}(\lambda) e_{-}(\lambda) d \lambda-\frac{\gamma}{2} \int_{-\infty}^{+\infty} f_{-}(\lambda) e_{+}(\lambda) d \lambda, \\
& =-\frac{1}{2}\left(B_{-+}+B_{+-}\right),
\end{aligned}
$$

which due to $B_{-+}=B_{+-}$means that

$$
\partial_{x} \sigma=-B_{+-} .
$$

proving the first part of Eq. (32). The second part is obtained differentiating once more with respect to $x$ and using Eq. (27).

The derivation of the differential equation (33) involving the $\beta$ derivative is more cumbersome. Again, the computations are similar with the impenetrable bosons case and we refer the reader to Chap. XIV of [3] .

The last differential equation (34) is obtained by replacing the potentials with $B_{+-}=-\partial_{x} \sigma$ and $B_{++}=\left(-\partial_{x}^{2} \sigma\right)^{1 / 2}$ in the R.H.S. of Eq. (33). It is also straightforward to see that $K_{T}(\lambda, \mu) \rightarrow 0$ when $x \rightarrow 0$ or $\beta \rightarrow-\infty$, which means that $\sigma=\ln \operatorname{det}\left(1-\gamma \hat{K}_{T}\right)=0$ in these limits. The first condition in (35) follows then from Eq. (28). This concludes the proof.

Finally, we end this section with a simple but important observation. Integrating equation $\partial_{x} \sigma=-B_{+-}$with initial conditions (35), we express the function $g(x, \beta, \gamma)$ (20) and, therefore, the field correlator (13), directly in terms of the potentials $B_{++}, B_{+-}$:

$$
g(x, \beta, \gamma)=B_{++}(x, \beta, \gamma) e^{-\int_{0}^{x} B_{+-}(y, \beta, \gamma) d y} .
$$

This expression will be used in our subsequent analysis of the field correlator.

\section{E. The zero-temperature limit}

In the zero temperature limit, the field-field correlator (13) depends only on three variables, the distance $\left(x_{1}-x_{2}\right)>$ 0 , the Fermi momentum $q=\sqrt{h}$ (or, equivalently, the chemical potential $h$ ), and the statistics parameter $\kappa$. The distance and momentum dependence can be encoded by one variable:

$$
\xi=\frac{\left(x_{1}-x_{2}\right)}{2} q=x \beta^{1 / 2},
$$


where now the rescaled variables are $x=\left(x_{1}-x_{2}\right) / 2$ and $\beta=h$. At $T=0$, the integral operator $\hat{K}$ acts on the interval $[-q, q]$ and has the kernel $K(\lambda, \mu)=\sin x(\lambda-\mu) /(\lambda-\mu)$. The logarithm of the determinant is

$$
\tilde{\sigma}_{0}(\xi, \gamma)=\left.\ln \operatorname{det}(1-\gamma \hat{K})\right|_{\gamma=\left(1+e^{i \pi \kappa}\right) / \pi} .
$$

The partial differential equation (34) characterizing $\sigma(x, \beta, \gamma)$ becomes then an ordinary differential equation in $\xi$. Introducing the new function

$$
\sigma_{0}=\xi\left(\tilde{\sigma}_{0}\right)^{\prime}
$$

where prime denotes the derivative with respect to $\xi$, we transform Eq. (34) into

$$
\left(\xi \sigma_{0}^{\prime \prime}\right)^{2}+4\left(\xi \sigma_{0}^{\prime}-\sigma_{0}\right)\left[4 \xi \sigma_{0}-4 \sigma_{0}+\left(\sigma_{0}^{\prime}\right)^{2}\right]=0
$$

with the boundary conditions

$$
\sigma_{0}=-2 \gamma \xi-4 \gamma^{2} \xi^{2}+O\left(\xi^{3}\right), \quad \gamma=\left(1+e^{i \pi \kappa}\right) / \pi .
$$

The ordinary differential equation (39) is the same Painlevé V equation obtained by Jimbo, Miwa, Môri and Sato in their celebrated work on the one-particle reduced density matrix (field-field correlator) of impenetrable bosons [30]. The only difference we find in the anyonic case is the boundary conditions (40) which, unlike the differential equation itself, depend on the statistics parameter. In a certain sense, this result could be expected, since it was noticed already by Jimbo et al. that the same equation (39), but with different boundary conditions, characterizes the density matrix of both the impenetrable bosons and the free fermions. Similar situation was also noted by Forrester, Frankel, Garoni and Witte [35] in their study of systems of finite number of particles with periodic boundary conditions. In this case, the reduced density matrix satisfies a Painlevé VI differential equation. Their work was recently extended to impenetrable anyons by Santachiara and Calabrese [34]. At $T=0$, our results agree with those of Santachiara and Calabrese. Indeed, Eq. (A5) for the first terms of the short-distance expansion of the field-field correlator, which reflects the boundary conditions (40), reduces at $T=0$ to:

$$
\left\langle\Psi^{\dagger}\left(x_{1}\right) \Psi\left(x_{2}\right)\right\rangle_{0}=D_{0}\left(1-\frac{\pi^{2}}{6} D_{0}^{2}\left(x_{1}-x_{2}\right)^{2}+\gamma \frac{\pi^{3}}{18} D_{0}^{3}\left(x_{1}-x_{2}\right)^{3}\right)+O\left(\left(x_{1}-x_{2}\right)^{4}\right), \quad \gamma=\left(1+e^{i \pi \kappa}\right) / \pi,
$$

where $D_{0}=q / \pi$ is the density at $T=0$. This result coincides with the expansion obtained in [34] - see Eq. (47) of that work and note that the definition of the statistical parameter $\kappa$ there differs in sign from ours.

\section{THE MATRIX RIEMANN-HILBERT PROBLEM FOR THE FIELD-FIELD CORELATOR}

The most difficult step in the analysis of the field-field correlation function is the calculation of its large-distance asymptotics. As we have seen in the previous sections, the correlation function is characterized by a completely integrable system of nonlinear partial differential equations - see Thm. [II.1, (13) and (37). A powerful method of analyzing these differential equations is the matrix Riemann-Hilbert problem (RHP) formalism [38]. The solution of the associated matrix RHP will allow us to obtain the large-distance asymptotics of the potentials $B_{+-}, B_{++}$, and therefore, the large-distance asymptotics of the correlator.

We start by formulating the matrix Riemann-Hilbert problem relevant to the case of potentials $B_{+-}, B_{++}$. In what follows, $\kappa \in(0,1]$. As we will see below, in this case we need to find a $2 \times 2$ matrix-valued function $\chi(\lambda)$, nonsingular for all $\lambda \in \mathbb{C}$ and analytic separately in the upper and lower half-plane, which is equal to the unit matrix at $\lambda=\infty$

$$
\chi(\infty)=I,
$$

and has the boundary values on the real axis which satisfy the condition

$$
\chi_{-}(\lambda)=\chi_{+}(\lambda) G(\lambda), \quad \chi_{ \pm}(\lambda)=\lim _{\epsilon \rightarrow 0^{+}} \chi(\lambda \pm i \epsilon) \quad \lambda \in \mathbb{R} .
$$

The matrix $G(\lambda)$ is called the conjugation matrix associated with the RHP and is defined only for real $\lambda$. In our case, it has the form

$$
G(\lambda)=\left(\begin{array}{cc}
1+\pi \gamma e_{+}(\lambda) e_{-}(\lambda), & -\pi \gamma e_{+}^{2}(\lambda) \\
\pi \gamma e_{-}^{2}(\lambda), & 1-\pi \gamma e_{+}(\lambda) e_{-}(\lambda)
\end{array}\right)
$$

where all notations are the same as before, e.g., $e_{ \pm}(\lambda)=\sqrt{\vartheta(\lambda)} e^{ \pm i \lambda x}$, etc. This means that both $G(\lambda)$ and $\chi(\lambda)$ depend also on $x, \beta$ and $\kappa$, but this dependence will be suppressed in our notations. 


\section{A. Relation to the auxiliary potentials}

In this section, we show how the auxiliary potentials $B_{+-}, B_{++}$are obtained from the solution of the RHP (42). The normalization condition $\chi(\infty)=I$ means that the solution of the RHP has the following expansion for large $\lambda$

$$
\chi(\lambda)=I+\frac{\Psi_{1}}{\lambda}+O\left(\frac{1}{\lambda^{2}}\right),
$$

where $\Psi_{1}(x, \beta)$ is a $2 \times 2$ matrix which depends only on $\beta$ and $x$. Then, the following symmetry of the conjugation matrix

$$
G(\lambda)=\sigma_{1} G^{-1}(-\lambda) \sigma_{1}
$$

that can be checked explicitly from the definition (43), determines the structure of $\Psi_{1}$. This matrix can be written as

$$
\Psi_{1}=\frac{1}{2 i}\left(\begin{array}{cc}
B_{+-} & -B_{++} \\
B_{++} & -B_{+-}
\end{array}\right)
$$

where the factor $1 / 2 i$ is introduced for convenience, and at the moment we do not know yet that $B_{++}$and $B_{+-}$in Eq. (46) are the auxiliary potentials (19). To prove this, we use the following formulation of the RHP. One can show directly (see, e.g, Chap. XV of [3] ) that the matrix RHP is equivalent to the system of singular integral equations:

$$
\chi_{+}(\lambda)=I+\frac{1}{2 \pi i} \int_{-\infty}^{+\infty} \frac{\chi_{+}(\mu)[I-G(\mu)]}{\mu-\lambda-i 0} d \mu, \quad \lambda \in \mathbb{R} .
$$

If we define

$$
\tilde{\chi}(\lambda)=\chi_{+}(\lambda) E(\lambda)
$$

where $E(\lambda)$ is the triangular matrix

$$
E(\lambda)=\left(\begin{array}{ll}
1 & e_{+}(\lambda) \\
0 & e_{-}(\lambda)
\end{array}\right)
$$

the system of integral equations (47) can be rewritten as

$$
\tilde{\chi}(\lambda)=E(\lambda)+\frac{1}{2 \pi i} \int_{-\infty}^{+\infty} \frac{\tilde{\chi}(\mu) \tilde{G}(\mu, \lambda)}{\mu-\lambda-i 0} d \mu, \quad \lambda \in \mathbb{R},
$$

with

$$
\tilde{G}(\lambda, \mu)=E^{-1}(\mu)[I-G(\mu)] E(\lambda)=\left(\begin{array}{cc}
0 & 0 \\
-\pi \gamma e_{-}(\mu) & \pi \gamma\left(e_{+}(\mu) e_{-}(\lambda)-e_{+}(\lambda) e_{-}(\mu)\right)
\end{array}\right) .
$$

For the matrix elements, Eq. (49) gives the integral equations:

$$
\begin{aligned}
& \tilde{\chi}_{12}=e_{+}(\lambda)+\gamma \int_{-\infty}^{+\infty} K_{T}(\lambda, \mu) \tilde{\chi}_{12}(\mu) d \mu, \\
& \tilde{\chi}_{22}=e_{-}(\lambda)+\gamma \int_{-\infty}^{+\infty} K_{T}(\lambda, \mu) \tilde{\chi}_{22}(\mu) d \mu,
\end{aligned}
$$

with the same kernel (15) we considered before:

$$
K_{T}(\lambda, \mu)=\frac{e_{+}(\lambda) e_{-}(\mu)-e_{-}(\lambda) e_{+}(\mu)}{2 i(\lambda-\mu)},
$$

and

$$
\begin{aligned}
& \tilde{\chi}_{11}(\lambda)=1-\frac{\gamma}{2 i} \int_{-\infty}^{+\infty} \frac{\tilde{\chi}_{12}(\mu) e_{-}(\mu)}{\mu-\lambda-i 0} d \mu \\
& \tilde{\chi}_{21}(\lambda)=-\frac{\gamma}{2 i} \int_{-\infty}^{+\infty} \frac{\tilde{\chi}_{22}(\mu) e_{+}(\mu)}{\mu-\lambda-i 0} d \mu
\end{aligned}
$$


In the first two equations, the nonsingular character of the kernel $K_{T}$ makes it possible to neglect the infinitesimal shift in the denominator. These equations are just the integral equations (17) defining $f_{ \pm}(\lambda)$. Therefore, we can make the following identification

$$
\tilde{\chi}_{12}(\lambda)=f_{+}(\lambda), \quad \tilde{\chi}_{22}(\lambda)=f_{-}(\lambda)
$$

Also, noting from Eq. (48) that $\tilde{\chi}_{11}(\lambda)=\chi_{11,+}(\lambda)$ and $\tilde{\chi}_{21}(\lambda)=\chi_{22,+}(\lambda)$, and are analytical in the upper half plane of $\lambda$, we have

$$
\begin{aligned}
& \chi_{11}(\lambda)=1-\frac{\gamma}{2 i} \int_{-\infty}^{+\infty} \frac{f_{+}(\mu) e_{-}(\mu)}{\mu-\lambda} d \mu, \quad \Im \lambda>0, \\
& \chi_{21}(\lambda)=-\frac{\gamma}{2 i} \int_{-\infty}^{+\infty} \frac{f_{-}(\mu) e_{-}(\mu)}{\mu-\lambda} d \mu, \quad \Im \lambda>0 .
\end{aligned}
$$

Taking the limit $\lambda \rightarrow \infty$ in these equations, we obtain

$$
\begin{aligned}
& \chi_{11}(\lambda)=1+\frac{\gamma}{2 i \lambda} \int_{-\infty}^{+\infty} f_{+}(\mu) e_{-}(\mu) d \mu+O\left(\frac{1}{\lambda^{2}}\right), \\
& \chi_{21}(\lambda)=\frac{\gamma}{2 i \lambda} \int_{-\infty}^{+\infty} f_{-}(\mu) e_{-}(\mu) d \mu+O\left(\frac{1}{\lambda^{2}}\right) .
\end{aligned}
$$

which shows that the components of the matrix $\Psi_{1}$ are indeed the potentials (19), as assumed in Eq. (46). As a byproduct of this derivation, one can also see from Eq. (50) that a part of the matrix product (48) means that

$$
\left(\begin{array}{c}
f_{+}(\lambda) \\
f_{-}(\lambda)
\end{array}\right)=\chi_{+}(\lambda)\left(\begin{array}{c}
e_{+}(\lambda) \\
e_{-}(\lambda)
\end{array}\right)
$$

This relation will be used in Sec. $\mathrm{V}$ in the analysis of the large-distance asymptotics.

\section{B. An useful transformation of the RHP}

As we have shown above, the potentials $B_{++}, B_{+-}$that completely characterize the field-field correlator (13) through Eq. (37), can be obtained from the expansion of the solution of the matrix RHP (42). This means that the large-distance asymptotics of the potentials, and hence of the field correlator, can be obtained from the solution of the RHP. To find this solution, we will use equivalent formulation of the RHP in terms of the singular integral equation (47). As a first step, we transform the matrix RHP (42) into a new form which has conjugation matrix with 1 on the diagonal. This is achieved by introducing the matrix function $\Phi$ defined as

$$
\Phi(\lambda)=\chi(\lambda)\left(\begin{array}{cc}
\beta^{-1}(\lambda) & 0 \\
0 & \alpha^{-1}(\lambda)
\end{array}\right)
$$

where the functions $\alpha(\lambda), \beta(\lambda)$ are:

$$
\alpha(\lambda)=\exp \left\{-\frac{1}{2 \pi i} \int_{-\infty}^{+\infty} \frac{d \mu}{\mu-\lambda} \ln \left(\frac{e^{\mu^{2}-\beta}-e^{i \pi \kappa}}{e^{\mu^{2}-\beta}+1}\right)\right\},
$$

and

$$
\beta(\lambda)=\exp \left\{-\frac{1}{2 \pi i} \int_{-\infty}^{+\infty} \frac{d \mu}{\mu-\lambda} \ln \left(\frac{e^{\mu^{2}-\beta}+e^{i \pi \kappa}+2}{e^{\mu^{2}-\beta}+1}\right)\right\} .
$$

The branch of the logarithm in these equations is specified by the requirement that $\ln (\ldots) \rightarrow 0$ for $\mu \rightarrow \infty$, and it is important at this point that $\kappa \in(0,1]$. Both functions $\alpha(\lambda)$ and $\beta(\lambda)$ are analytic separately in the upper and lower half-plane, and are the solutions of the following scalar Riemann-Hilbert problems (see Appendix D)

$$
\alpha_{-}(\lambda)=\alpha_{+}(\lambda) g_{\alpha}(\lambda), \lambda \in \mathbb{R} ; \quad \alpha(\infty)=1 ; \quad g_{\alpha}(\lambda)=\frac{e^{\lambda^{2}-\beta}-e^{i \pi \kappa}}{e^{\lambda^{2}-\beta}+1}
$$


and

$$
\beta_{-}(\lambda)=\beta_{+}(\lambda) g_{\beta}(\lambda), \lambda \in \mathbb{R} ; \quad \beta(\infty)=1 ; \quad g_{\beta}(\lambda)=\frac{e^{\mu^{2}-\beta}+e^{i \pi \kappa}+2}{e^{\mu^{2}-\beta}+1} .
$$

They also have the property

$$
\alpha^{-1}(\lambda)=\alpha(-\lambda), \quad \beta^{-1}(\lambda)=\beta(-\lambda) .
$$

Using these relations one can see from Eq. (42) that the matrix $\Phi$ satisfies the following RHP

$$
\Phi_{-}(\lambda)=\Phi_{+}(\lambda) G_{\Phi}(\lambda), \quad \lambda \in \mathbb{R} ; \quad \Phi(\infty)=1,
$$

with the conjugation matrix

$$
G_{\Phi}(\lambda)=\left(\begin{array}{cc}
1 & -\pi \gamma \vartheta(\lambda) \beta_{+}(\lambda) \alpha_{-}^{-1}(\lambda) e^{2 i \lambda x} \\
\pi \gamma \vartheta(\lambda) \alpha_{+}(\lambda) \beta_{-}^{-1}(\lambda) e^{-2 i \lambda x} & 1
\end{array}\right) .
$$

Equations (44) and (46), together with the fact that $\alpha(\lambda), \beta(\lambda) \rightarrow 1$ at $\lambda \rightarrow \infty$, show that in terms of the components of the matrix $\Phi$ that solves the transformed RHP (56), the potentials $B_{++}, B_{-+}$are expressed as

$$
B_{+-}=2 i \lim _{\lambda \rightarrow \infty} \lambda\left[\Phi_{11}(\lambda)-\beta^{-1}(\lambda)\right], \quad B_{++}=-2 i \lim _{\lambda \rightarrow \infty} \lambda \Phi_{12}(\lambda) .
$$

The RHP (56) and formulae (58) will be the basis for our analysis of the large-distance asymptotics of the field-field correlator of impenetrable anyons.

\section{LARGE-DISTANCE ASYMPTOTIC ANALYSIS}

In this section, we perform the large-distance asymptotic analysis of the RHP (56). The strategy is to use Eq. (58) to obtain the auxiliary potentials $B_{++}(x, \beta, \kappa)$ and $B_{+-}(x, \beta, \kappa)$ from the large- $\lambda$ expansion of $\Phi_{11,+}(\lambda)$ and $\Phi_{12,+}(\lambda)$. Then, the function $\sigma(x, \beta, \kappa)$ can be calculated using the differential equations (32) and (33).

\section{A. The auxiliary potentials in the large- $x$ limit}

The fact that the conjugation matrix $G_{\Phi}$ of the RHP (56) has 1 on the diagonal simplifies the matrix structure of the kernel $I-G_{\Phi}$ of the integral equation formulation (47) of this problem. Combined with the explicit form (57) of the off-diagonal elements of $G_{\Phi}$, and Eqs. (54) and (55) for $\alpha$ and $\beta$, the matrix integral equation gives then the following equations for $\Phi_{11,+}(\lambda), \Phi_{12,+}(\lambda)$ :

$$
\Phi_{11,+}(\lambda)=1-\frac{\left(1+e^{i \pi \kappa}\right)}{2 \pi i} \int_{-\infty}^{+\infty} \frac{\Phi_{12,+}(\mu)}{(\mu-\lambda-i 0)} \frac{\alpha_{-}(\mu)}{\beta_{-}(\mu)} \frac{e^{-2 i \mu x}}{\left(e^{\mu^{2}-\beta}-e^{i \pi \kappa}\right)}, \quad \Im \lambda=0,
$$

and

$$
\Phi_{12,+}(\lambda)=\frac{\left(1+e^{i \pi \kappa}\right)}{2 \pi i} \int_{-\infty}^{+\infty} \frac{\Phi_{11,+}(\mu)}{(\mu-\lambda-i 0)} \frac{\beta_{+}(\mu)}{\alpha_{+}(\mu)} \frac{e^{2 i \mu x}}{\left(e^{\mu^{2}-\beta}-e^{i \pi \kappa}\right)}, \quad \Im \lambda=0 .
$$

An important feature of these equations is that the analytical properties of $\alpha(\lambda)$ and $\beta(\lambda)$ together with the fact that $\Phi_{11}(\infty)=\alpha(\infty)=\beta(\infty)=1$, make it possible to obtain an estimate of $\Phi_{12,+}$ by shifting the contour in the upper half-plane and evaluating the integral by the sum of the residues. Strictly speaking, this approach requires the functions $\Phi$ to conform to estimates of the type $\left|\Phi_{12}(\lambda, x, \beta)\right| \leq C / \lambda$ and $\left|\Phi_{11}(\lambda, \beta, x)-1\right| \leq D / \lambda$ for $\Im \lambda \geq 0$ in some ranges of $\beta$ and $x$ : $\beta<\beta_{0}, x>x_{0}$, where $C, D$ depends only on the constants $\beta_{0}, x_{0}$. In what follows, we assume that these estimates hold, noting that they can be justified self-consistently as in Sec. 4 of [5]. The poles of the integrand in Eq. (60) are given by $\lambda+i 0$ and the zeros $\lambda_{k}$ of the function $e^{\lambda^{2}-\beta}-e^{i \pi \kappa}$ in the upper half-plane. The zeros of this function are given by the formulae:

$$
\left(\Re \lambda_{k}\right)^{2}=\frac{1}{2}\left(\beta+\sqrt{\beta^{2}+\pi^{2}[\kappa+2 k]^{2}}\right), \quad\left(\Im \lambda_{k}\right)^{2}=\frac{1}{2}\left(-\beta+\sqrt{\beta^{2}+\pi^{2}[\kappa+2 k]^{2}}\right), \quad k=0, \pm 1, \pm 2, \cdots,
$$


and, as we will see below, the leading terms in the asymptotic behavior are determined by $\lambda_{0}^{+}$and $\lambda_{-1}^{+}$which are closest to the real axis:

$$
\lambda_{0}^{+}=\left(\beta+\sqrt{\beta^{2}+\pi^{2} \kappa^{2}}\right)^{1 / 2} / \sqrt{2}+i\left(-\beta+\sqrt{\beta^{2}+\pi^{2} \kappa^{2}}\right)^{1 / 2} / \sqrt{2},
$$

and

$$
\lambda_{-1}^{+}=-\left(\beta+\sqrt{\beta^{2}+\pi^{2}[\kappa-2]^{2}}\right)^{1 / 2} / \sqrt{2}+i\left(-\beta+\sqrt{\beta^{2}+\pi^{2}[\kappa-2]^{2}}\right)^{1 / 2} / \sqrt{2} .
$$

The superscript + denotes the zeros in the upper half-plane. Closing the contour in this half-plane, we obtain

$$
\Phi_{12,+}(\lambda)=\left(1+e^{i \pi \kappa}\right) \Phi_{11,+}(\lambda) \frac{\beta_{+}(\lambda)}{\alpha_{+}(\lambda)} \frac{e^{2 i \lambda x}}{\left(e^{\lambda^{2}-\beta}-e^{i \pi \kappa}\right)}+S^{+}(\lambda),
$$

with

$$
S^{+}(\lambda)=\sum_{k=-\infty}^{+\infty} \frac{\left(1+e^{i \pi \kappa}\right)}{2 e^{i \pi \kappa}} \frac{\beta\left(\lambda_{k}^{+}\right)}{\alpha\left(\lambda_{k}^{+}\right)} \frac{\Phi_{11}\left(\lambda_{k}^{+}\right) e^{2 i \lambda_{k}^{+} x}}{\left(\lambda_{k}^{+}-\lambda\right) \lambda_{k}^{+}}
$$

The series $S^{+}(\lambda)$ is uniformly convergent for $\lambda \in \mathbb{R}, x_{0} \leq x, 0<\kappa \leq 1$, and with $\beta \leq \beta_{0}$. Substituting Eq. 64) into (59), and using the relations (54) and (55), we obtain the following representation for Eq. (59):

$$
\begin{gathered}
\Phi_{11,+}(\lambda)=1-\frac{\left(1+e^{i \pi \kappa}\right)^{2}}{2 \pi i} \int_{-\infty}^{+\infty} \frac{\Phi_{11,+}(\mu)}{\mu-\lambda-i 0} \frac{d \mu}{\left(e^{\mu^{2}-\beta}-e^{i \pi \kappa}\right)\left(e^{\mu^{2}-\beta}+e^{i \pi \kappa}+2\right)} \\
-\frac{1}{2 \pi i} \int_{-\infty}^{+\infty} \frac{R^{+}(\mu)}{\mu-\lambda-i 0} d \mu,
\end{gathered}
$$

where

$$
R^{+}(\mu)=\left(1+e^{i \pi \kappa}\right) \frac{\alpha_{-}(\mu)}{\beta_{-}(\mu)} \frac{e^{-2 i \mu x}}{\left(e^{\mu^{2}-\beta}-e^{i \pi \kappa}\right)} S^{+}(\mu) .
$$

The form of the integral equation (65) suggests (see Eq. (D5) in Appendix D) that it is useful to consider the following inhomogeneous scalar RH problem for the function $\tilde{\Phi}(\lambda)$ :

$$
\tilde{\Phi}_{-}(\lambda)=\tilde{\Phi}_{+}(\lambda) g(\lambda)+R^{+}(\lambda), \quad \lambda \in \mathbb{R}, \quad \tilde{\Phi}(\infty)=1,
$$

such that $\tilde{\Phi}_{+}(\lambda)=\Phi_{11,+}(\lambda)$ for $\lambda \in \mathbb{R}$. The function $g$ in this RHP is given by the relation

$$
1-g(\lambda)=-\frac{\left(1+e^{i \pi \kappa}\right)^{2}}{\left(e^{\lambda^{2}-\beta}-e^{i \pi \kappa}\right)\left(e^{\lambda^{2}-\beta}+e^{i \pi \kappa}+2\right)},
$$

which shows that using Eqs. (54) and (55), $g(\lambda)$ can be written as

$$
g(\lambda)=\frac{\left(e^{\lambda^{2}-\beta}+1\right)^{2}}{\left(e^{\lambda^{2}-\beta}-e^{i \pi \kappa}\right)\left(e^{\lambda^{2}-\beta}+e^{i \pi \kappa}+2\right)}=\frac{\alpha_{-}^{-1}(\lambda) \beta_{-}^{-1}(\lambda)}{\alpha_{+}^{-1}(\lambda) \beta_{+}^{-1}(\lambda)} .
$$

This form of $g(\lambda)$ combined with Eq. (D4) implies that the solution of the RH problem (66) is:

$$
\tilde{\Phi}(\lambda)=\alpha^{-1}(\lambda) \beta^{-1}(\lambda)-\frac{\alpha^{-1}(\lambda) \beta^{-1}(\lambda)}{2 \pi i} \int_{-\infty}^{+\infty} \frac{\alpha_{-}(\mu) \beta_{-}(\mu) R(\mu)}{\mu-\lambda} d \mu, \quad \lambda \in \mathbb{C} / \mathbb{R} .
$$

The functions $\tilde{\Phi}(\lambda)$ and $\Phi_{11}(\lambda)$ are analytic in the upper half-plane. They have the same behavior at infinity: $\tilde{\Phi}(\infty)=\Phi_{11}(\infty)=1$, and their boundary values at the real axis $\tilde{\Phi}_{+}(\lambda), \Phi_{11,+}(\lambda), \lambda \in \mathbb{R}$ are equal. This means that they coincide, $\Phi_{11}(\lambda)=\tilde{\Phi}(\lambda)$, for $\Im \lambda>0$. Therefore, from Eq. (67) and the explicit expression for $R^{+}(\lambda)$ we have

$$
\Phi_{11}(\lambda)=\alpha^{-1}(\lambda) \beta^{-1}(\lambda)-\frac{\alpha^{-1}(\lambda) \beta^{-1}(\lambda)}{2 \pi i} \sum_{k=-\infty}^{+\infty} A\left(\lambda_{k}^{+}, \lambda\right) \Phi_{11}\left(\lambda_{k}^{+}\right), \quad \Im \lambda \geq 0,
$$


where

$$
A\left(\lambda_{k}^{+}, \lambda\right)=\frac{\left(1+e^{i \pi \kappa}\right)^{2}}{2 \lambda_{k}^{+} e^{i \pi \kappa}} \frac{\beta\left(\lambda_{k}^{+}\right)}{\alpha\left(\lambda_{k}^{+}\right)} e^{2 i \lambda_{k}^{+} x} \int_{-\infty}^{+\infty} \frac{\alpha_{-}^{2}(\mu) e^{-2 i \mu x}}{\left(e^{\mu^{2}-\beta}-e^{i \pi \kappa}\right)\left(\lambda_{k}^{+}-\mu\right)(\mu-\lambda)} d \mu
$$

For $x \rightarrow \infty$, the integral in the last equation can be estimated as $C\left|e^{-2 i \lambda_{0}^{-} x}\right|$, where $\lambda_{0}^{-}$is the $k=0$ zero (61) in the lower half-plane, so the leading term of $\Phi_{11}$ in the limit $x \rightarrow \infty$ is given by

$$
\Phi_{11}(\lambda)=\alpha^{-1}(\lambda) \beta^{-1}(\lambda)+O\left(e^{-4 \Im \lambda_{0}^{+} x}\right), \quad \Im \lambda>0,
$$

where we took into account that the magnitudes of the imaginary parts of $\lambda_{0}^{ \pm}$are the same. Using Eq. (68) in (60), we obtain the leading term of $\Phi_{12}$ for large $x$ :

$$
\Phi_{12}(\lambda)=\frac{\left(1+e^{i \pi \kappa}\right)}{2 \pi i} \int_{-\infty}^{+\infty} \frac{\alpha_{+}^{-2}(\mu)}{(\mu-\lambda-i 0)} \frac{e^{2 i \mu x}}{\left(e^{\mu^{2}-\beta}-e^{i \pi \kappa}\right)}+O\left(e^{-4 \Im \lambda_{0}^{+} x}\right), \quad \Im \lambda>0 .
$$

Substitution of the asymptotics (68) and (69) into Eq. (58) gives the large-distance asymptotic behavior of the potentials $B_{++}, B_{+-}$:

$$
B_{+-}=\frac{1}{\pi} \int_{-\infty}^{+\infty} \ln \left(\frac{e^{\mu^{2}-\beta}+1}{e^{\mu^{2}-\beta}-e^{i \pi \kappa}}\right) d \mu+O\left(e^{-4 \Im \lambda_{0}^{+} x}\right), \quad x \rightarrow \infty
$$

where the branch of the logarithm is fixed by the requirement that $\ln (\ldots) \rightarrow 0$ for $\mu \rightarrow \infty$, and

$$
B_{++}=\frac{\left(1+e^{i \pi \kappa}\right)}{\pi} \int_{-\infty}^{+\infty} \frac{\alpha_{+}^{-2}(\mu) e^{2 i \mu x}}{\left(e^{\mu^{2}-\beta}-e^{i \pi \kappa}\right)} d \mu+O\left(e^{-4 \Im \lambda_{0}^{+} x}\right), \quad x \rightarrow \infty .
$$

The expression for $B_{++}$can be made more precise by closing the contour in the upper half-plane and evaluating the integral as the sum of the residues

$$
B_{++}=i\left(1+e^{-i \pi \kappa}\right) \sum_{k=\text { min }}^{\max } \frac{\alpha^{-2}\left(\lambda_{k}^{+}\right)}{\lambda_{k}^{+}} e^{2 i \lambda_{k}^{+} x}+O\left(e^{-4 \Im \lambda_{0}^{+} x}\right),
$$

where the accuracy of our calculation of the leading term in the asymptotics implies that the sum over poles can be limited to the interval between $k=\min (\beta, \kappa)<0$ and $k=\max (\beta, \kappa)>0$ that are defined by the relations

$$
\Im \lambda_{\min }^{+}<2 \Im \lambda_{0}^{+}, \Im \lambda_{\min -1}^{+}>2 \Im \lambda_{0}^{+},
$$

and

$$
\Im \lambda_{\max }^{+}<2 \Im \lambda_{0}^{+}, \quad \Im \lambda_{\max +1}^{+}>2 \Im \lambda_{0}^{+} .
$$

\section{B. Determination of $\sigma(x, \beta, \kappa)$ in the large- $x$ limit}

The large-distance asymptotics of the auxiliary potentials obtained in the previous section and the differential equations (32) and (33) give the following asymptotic behavior of the function $\sigma(x, \beta, \gamma) \equiv \ln \operatorname{det}\left(1-\gamma \hat{K}_{T}\right)$ :

$$
\sigma(x, \beta, \kappa)=-x C(\beta, \kappa)+c(\beta, \kappa)+O\left(e^{-4 \Im \lambda_{0}^{+} x}\right), x \rightarrow \infty,
$$

where $C(\beta, \kappa)$ is defined by Eq. (3), and $c(\beta, \kappa)$ is a constant that depends on $\beta$ and $\kappa$, but is independent of $x$. In order to determine this constant, which through $\sigma(x, \beta, \kappa)$ determines the $\beta$ - and $\kappa$-dependence of the amplitudes of the asymptotic terms in the expansion of the field correlators, we integrate the relation

$$
\partial_{\gamma} \sigma=-\int_{-\infty}^{+\infty} R_{T}(\lambda, \lambda) d \lambda
$$

which is obtained by taking the derivative of Eq. (36) with respect to $\gamma$ and using the relation $\left(1-\gamma \hat{K}_{T}\right)^{-1} \hat{K}_{T}=\hat{R}_{T}$. As shown in Sec. ЩIC, $R_{T}(\lambda, \lambda)$ in Eq. (75) is:

$$
R_{T}(\lambda, \lambda)=\frac{1}{2 i}\left(\partial_{\lambda} f_{+}(\lambda) f_{-}(\lambda)-f_{+}(\lambda) \partial_{\lambda} f_{-}(\lambda)\right) .
$$


Our approach is similar to the one employed in Ref. [36] (see also 26]), where the authors considered a generalized sine-kernel at zero temperature and studied the large-distance asymptotic behavior of the Fredholm determinant using the nonlinear steepest descent method of Deift and Zhou [37]. At zero temperature, $\operatorname{det}(1-\gamma \hat{K})$ becomes a particular case of the Fredholm determinant considered in [36].

The first step is the computation of $f_{ \pm}(\lambda)$ using the relation (52) and the following expression for the function $\chi(\lambda)$ in this relation:

$$
\chi(\lambda)=\Phi(\lambda)\left(\begin{array}{lr}
\beta(\lambda) & 0 \\
0 & \alpha(\lambda)
\end{array}\right) .
$$

In the previous Section, we have obtained Eqs. (68) and (69) for the matrix elements $\Phi_{11}(\lambda)$ and $\Phi_{12}(\lambda)$ of the matrix $\Phi(\lambda)$ in the large- $x$ limit. Closing the integration contour in the upper half plane in Eq. (69), one gets more explicitly:

$$
\Phi_{12}(\lambda)=\pi \gamma \frac{\alpha_{+}^{-2}(\lambda)}{\left(e^{\lambda^{2}-\beta}-e^{i \pi \kappa}\right)} e^{2 i \lambda x}+O\left(e^{-2 \Im \lambda_{0}^{+} x}\right) .
$$

One needs to find similar asymptotics for $\Phi_{21}$ and $\Phi_{22}$. As in the previous Section, the integral formulation (47) of the RHP gives the following equations for these matrix elements:

$$
\Phi_{21,+}(\lambda)=-\frac{\gamma}{2 i} \int_{-\infty}^{+\infty} \frac{\Phi_{22,+}(\mu)}{\mu-\lambda-i 0} \frac{\alpha_{-}(\mu)}{\beta_{-}(\mu)} \frac{e^{-2 i \mu x}}{\left(e^{\mu^{2}-\beta}-e^{i \pi \kappa}\right)} d \mu, \quad \lambda \in \mathbb{R}
$$

and

$$
\Phi_{22,+}(\lambda)=1+\frac{\gamma}{2 i} \int_{-\infty}^{+\infty} \frac{\Phi_{21,+}(\mu)}{\mu-\lambda-i 0} \frac{\beta_{+}(\mu)}{\alpha_{+}(\mu)} \frac{e^{2 i \mu x}}{\left(e^{\mu^{2}-\beta}-e^{i \pi \kappa}\right)} d \mu, \quad \lambda \in \mathbb{R} .
$$

In the integral equation (79), one can close the contour in the lower half plane obtaining

$$
\Phi_{21,+}(\lambda)=-\pi \gamma \sum_{k=-\infty}^{\infty} \frac{\Phi_{22,+}\left(\lambda_{k}^{-}\right)}{\lambda_{k}^{-}-\lambda-i 0} \frac{\alpha_{-}\left(\lambda_{k}^{-}\right)}{\beta_{-}\left(\lambda_{k}^{-}\right)} \frac{e^{-2\left|\Im \lambda_{k}^{-}\right| x}}{2 \lambda_{k}^{-} e^{i \pi \kappa}},
$$

where $\lambda_{k}^{-}$are the zeroes of $e^{\mu^{2}-\beta}-e^{i \pi \kappa}$ in the lower half plane. Substituting this result in the integral equation for $\Phi_{22,+}$ and closing the contour in the upper half plane we find

$$
\Phi_{22,+}(\lambda)=1+O\left(e^{-2\left(\left|\Im \lambda_{0}^{-}\right|+\Im \lambda_{0}^{+}\right) x}\right),
$$

and, combining Eqs. (81) and (79),

$$
\Phi_{21,+}(\lambda)=O\left(e^{-2\left|\Im \lambda_{0}^{-}\right| x}\right) .
$$

Now we are able to compute $f_{ \pm}(\lambda)$ up to exponentially small corrections in $x$. Explicitly, Eqs. (52) and (77) give:

$$
\left(\begin{array}{c}
f_{+}(\lambda) \\
f_{-}(\lambda)
\end{array}\right)=\left(\begin{array}{l}
\Phi_{11,+}(\lambda) \beta_{+}(\lambda) e_{+}(\lambda)+\Phi_{12,+}(\lambda) \alpha_{+}(\lambda) e_{-}(\lambda) \\
\Phi_{21,+}(\lambda) \beta_{+}(\lambda) e_{+}(\lambda)+\Phi_{22,+}(\lambda) \alpha_{+}(\lambda) e_{-}(\lambda)
\end{array}\right)
$$

and, therefore, using Eqs. (68), (78), (81), and (82) we find:

$$
\left(\begin{array}{c}
f_{+}(\lambda) \\
f_{-}(\lambda)
\end{array}\right)=\left(\begin{array}{c}
\alpha_{+}^{-1}(\lambda) e_{+}(\lambda) e^{2 \pi i \nu(\lambda)} \\
\alpha_{+}(\lambda) e_{-}(\lambda)
\end{array}\right)
$$

The function $\nu(\lambda)$ here (we have suppressed the dependence on $\beta$ ) is defined in Eq. (11). One can see directly that $\nu(\lambda)$ can be written in terms of the rescaled Fermi weight $\theta(\lambda)=\left(1+e^{\lambda^{2}-\beta}\right)^{-1}$ as

$$
\nu(\lambda)=-\frac{1}{2 \pi i} \log (1-\pi \gamma \theta(\lambda))=\frac{1}{2 \pi i} \log \left(\frac{e^{\lambda^{2}-\beta}+1}{e^{\lambda^{2}-\beta}-e^{i \pi \kappa}}\right),
$$

and also that

$$
\alpha_{+}(\lambda)=\exp \left\{i \pi \nu(\lambda)+\text { P.V. } \int_{-\infty}^{+\infty} \frac{\nu(\mu)}{\mu-\lambda} d \mu\right\}
$$


Using Eqs. (83) and (76) in Eq. (75), we have

$$
\begin{aligned}
\partial_{\gamma} \sigma=-\int_{-\infty}^{+\infty} R_{T}(\lambda, \lambda) d \lambda & =-\int_{-\infty}^{+\infty} \frac{1}{2 \pi i} \frac{\pi \theta(\lambda)}{(1-\pi \gamma \theta(\lambda))}\left(2 i x-2 \partial_{\lambda} \log \alpha_{+}(\lambda)+2 i \pi \partial_{\lambda} \nu(\lambda)\right) d \lambda \\
& =-\int_{-\infty}^{+\infty} \partial_{\gamma} \nu(\lambda)\left(2 i x-2 \partial_{\lambda} \log \alpha_{+}(\lambda)+2 i \pi \partial_{\lambda} \nu(\lambda)\right) d \lambda
\end{aligned}
$$

Our current task is to write the RHS of Eq. (84) as a derivative with respect to $\gamma$. The term depending on $x$ already has this form, but the last two, $x$-independent, terms do not. We transform them as follows:

$$
\begin{aligned}
\int_{-\infty}^{+\infty} \partial_{\gamma} \nu(\lambda)\left(2 \partial_{\lambda} \log \alpha_{+}(\lambda)-2 i \pi \partial_{\lambda} \nu(\lambda)\right) & =\int_{-\infty}^{+\infty} \partial_{\gamma} \nu(\lambda) \partial_{\lambda} \int_{-\infty}^{+\infty}\left(\frac{\nu(\mu)}{\mu-\lambda-i 0}+\frac{\nu(\mu)}{\mu-\lambda+i 0}\right) d \mu d \lambda \\
& =\int_{-\infty}^{+\infty} \partial_{\gamma} \nu(\lambda)\left(\frac{\nu(\mu)}{(\lambda-\mu-i 0)^{2}}+\frac{\nu(\mu)}{(\lambda-\mu+i 0)^{2}}\right) d \mu d \lambda
\end{aligned}
$$

Next, we show that this expression is equal to

$$
\partial_{\gamma} \int_{-\infty}^{+\infty} \frac{\partial_{\lambda} \nu(\lambda) \nu(\mu)-\nu(\lambda) \partial_{\mu}(\mu)}{2(\lambda-\mu)} d \lambda d \mu
$$

Indeed,

$$
\begin{array}{r}
\partial_{\gamma} \int_{-\infty}^{+\infty} \frac{\partial_{\lambda} \nu(\lambda) \nu(\mu)-\nu(\lambda) \partial_{\mu} \nu(\mu)}{2(\lambda-\mu)} d \lambda d \mu=\int_{-\infty}^{+\infty} \frac{\left[\left(\partial_{\gamma} \partial_{\lambda} \nu(\lambda)\right) \nu(\mu)+\partial_{\lambda} \nu(\lambda) \partial_{\gamma} \nu(\mu)\right]}{\lambda-\mu} d \lambda d \mu \\
=\frac{1}{2} \int_{-\infty}^{+\infty}\left[\left(\partial_{\gamma} \partial_{\lambda} \nu(\lambda)\right) \nu(\mu)+\partial_{\lambda} \nu(\lambda) \partial_{\gamma} \nu(\mu)\right]\left(\frac{1}{\lambda-\mu+i 0}+\frac{1}{\lambda-\mu-i 0}\right) d \lambda d \mu \\
=\frac{1}{2} \int_{-\infty}^{+\infty}\left[\partial_{\gamma} \nu(\lambda) \nu(\mu)+\nu(\lambda) \partial_{\gamma} \nu(\mu)\right]\left(\frac{1}{(\lambda-\mu+i 0)^{2}}+\frac{1}{(\lambda-\mu-i 0)^{2}}\right) d \lambda d \mu \\
=\int_{-\infty}^{+\infty} \partial_{\gamma} \nu(\lambda)\left(\frac{\nu(\mu)}{(\lambda-\mu-i 0)^{2}}+\frac{\nu(\mu)}{(\lambda-\mu+i 0)^{2}}\right) d \mu d \lambda
\end{array}
$$

Therefore,

$$
\partial_{\gamma} \sigma=-2 i x \partial_{\gamma} \int_{-\infty}^{+\infty} \nu(\lambda) d \lambda+\partial_{\gamma} \int_{-\infty}^{+\infty} \frac{\partial_{\lambda} \nu(\lambda) \nu(\mu)-\nu(\lambda) \partial_{\mu}(\mu)}{2(\lambda-\mu)} d \lambda d \mu
$$

Integrating this relation with respect to $\gamma$ and taking into account that $\sigma(\gamma=0)=0$, we obtain

$$
\sigma(x, \beta, \kappa)=-x C(\beta, \kappa)+\int_{-\infty}^{+\infty} \frac{\partial_{\lambda} \nu(\lambda) \nu(\mu)-\nu(\lambda) \partial_{\mu}(\mu)}{2(\lambda-\mu)} d \lambda d \mu .
$$

Comparison with Eq. (74) shows finally that

$$
c(\beta, \kappa)=\int_{-\infty}^{+\infty} \frac{\partial_{\lambda} \nu(\lambda) \nu(\mu)-\nu(\lambda) \partial_{\mu} \nu(\mu)}{2(\lambda-\mu)} d \lambda d \mu
$$

One can also obtain an alternative expression for the constant $c(\beta, \kappa)$ by a simpler calculation. Using again the differential equation (33) and expressions for the potentials (70) and (71), we have

$$
\sigma(x, \beta, \kappa)=-x C(\beta, \kappa)+\frac{1}{2} \int_{-\infty}^{\beta}\left(\frac{d C\left(\beta^{\prime}, \kappa\right)}{d \beta^{\prime}}\right)^{2} d \beta^{\prime}+c(\kappa)+O\left(e^{-4 \Im \lambda_{0}^{+} x}\right), x \rightarrow \infty
$$

where $c(\kappa)$ is a constant that depends only on $\kappa$. The initial condition $\sigma(x,-\infty, \kappa)=0$ (35) together with the fact that the asymptotic behavior of $\sigma(x, \beta, \kappa)$ is uniform in $\beta$, and $\kappa \in(0,1]$, imply that $c(\kappa)=0$. This gives the following expression for $c(\beta, \kappa)$ :

$$
c(\beta, \kappa)=\frac{1}{2} \int_{-\infty}^{\beta}\left(\frac{d C\left(\beta^{\prime}, \kappa\right)}{d \beta^{\prime}}\right)^{2} d \beta^{\prime} .
$$




\section{Large-distance asymptotic behavior of the field-field correlator}

As a reminder, we note that the anyonic field-field correlation function can be written combining Eqs. (13) and (20) as follows:

$$
\left\langle\Psi^{\dagger}\left(x_{1}\right) \Psi\left(x_{2}\right)\right\rangle_{T}=\frac{\sqrt{T}}{2 \pi \gamma} B_{++}(x, \beta, \kappa) e^{\sigma(x, \beta, \kappa)}, \quad \gamma=\left(1+e^{i \pi \kappa}\right) / \pi .
$$

Using in this equation the expressions obtained in the previous Section for the potential $B_{++}$and $\sigma$, and going back to the original variables related to rescaled ones through $x=x_{12} \sqrt{T} / 2$ and $\beta=h / T$, we arrive at our main result for the large-distance asymptotic behavior of the field-field correlator

$$
\left\langle\Psi^{\dagger}\left(x_{1}\right) \Psi\left(x_{2}\right)\right\rangle_{T}=e^{-x_{12} \frac{\sqrt{T}}{2} C(h / T, \kappa)} e^{c(h / T, \kappa)} \sum_{k=\text { min }}^{\max } c_{k} e^{i x_{12} \sqrt{T} \lambda_{k}^{+}}+O\left(e^{-2 \Im \lambda_{0}^{+} \sqrt{T} x_{12}}\right),
$$

where

$$
c_{k}=i \frac{e^{i \pi \kappa} \sqrt{T}}{2} \frac{\alpha^{-2}\left(\lambda_{k}^{+}\right)}{\lambda_{k}^{+}}
$$

and $\lambda_{k}^{+}$are the zeros (61) in the upper half-plane. In the asymptotics (88), the functions $C(\beta, \kappa), c(\beta, \kappa)$, and $\alpha(\lambda)$, are defined, respectively, by Eqs. (3), (44) or (87), and (53), and the limits of summation over $k$ are given by (72) and (73). The leading part of the asymptotics is the term $k=0$, but as we approach the free fermionic point $\kappa \rightarrow 1$, the $k=-1$ term also becomes relevant.

\section{ANALYSIS OF THE RESULTS}

As the last step, we check the validity of our main result in the appropriate limits. When the statistics parameter $\kappa \rightarrow 0$ we should reproduce the results obtained for impenetrable bosons in [3, 5, 33]. Even though in the largedistance analysis performed above we have not made any distinction between the cases of negative and positive chemical potential (or, equivalently, $\beta$ ), in what follows, we will see that in the bosonic limit, the asymptotic behavior of the corrrelators is fundamentally different in the two regions, as one would expect from [3, 5, 33. 3 . In the fermionic limit $\kappa \rightarrow 1$, our result for the field-field correlator of the anyons should reduce to the correlator of the free fermions. At low temperatures, $\beta \rightarrow \infty$, and positive chemical potential, the system becomes critical, and our result should agree with the predictions of the conformal field theory [10, 18].

\section{A. The bosonic limit}

In the bosonic limit $\kappa \rightarrow 0$, Eq. (3) gives in the case of positive and negative chemical potential, respectively,

$$
\lim _{\kappa \rightarrow 0} C(\beta, \kappa)=\frac{1}{\pi} \int_{-\infty}^{+\infty} \ln \left|\frac{e^{\lambda^{2}-\beta}+1}{e^{\lambda^{2}-\beta}-1}\right| d \lambda+2 i \sqrt{\beta}, \quad \beta>0,
$$

and

$$
\lim _{\kappa \rightarrow 0} C(\beta, \kappa)=\frac{1}{\pi} \int_{-\infty}^{+\infty} \ln \left(\frac{e^{\lambda^{2}-\beta}+1}{e^{\lambda^{2}-\beta}-1}\right) d \lambda, \quad \beta<0
$$

Also, we have from Eq. (62)

$$
\lambda_{0}^{+}=\sqrt{\beta}, \quad \beta>0 ; \quad \lambda_{0}^{+}=i \sqrt{|\beta|}, \quad \beta<0 .
$$

Introducing the function $C(\beta)$ :

$$
C(\beta) \equiv \frac{1}{\pi} \int_{-\infty}^{+\infty} \ln \left|\frac{e^{\lambda^{2}-\beta}+1}{e^{\lambda^{2}-\beta}-1}\right| d \lambda, \quad \beta=h / T
$$


and using Eq. (88) reduced according to the previous equations of this Section, we obtain the following asymptotic behavior of the correlation function at negative chemical potential

$$
\begin{gathered}
\left\langle\Psi^{\dagger}\left(x_{1}\right) \Psi\left(x_{2}\right)\right\rangle_{T} \simeq \frac{T}{2 \sqrt{|h|}} \exp \left[a(h / T)+\frac{1}{2} \int_{-\infty}^{h / T}\left(\frac{d C\left(\beta^{\prime}, \kappa\right)}{d \beta^{\prime}}\right)^{2} d \beta^{\prime}\right] e^{-x_{12}\left[\frac{\sqrt{T}}{2} C(h / T)+\sqrt{|h|}\right]}, \\
h<0, \quad x_{12} \equiv\left(x_{1}-x_{2}\right) \rightarrow \infty,
\end{gathered}
$$

where

$$
a(\beta)=\frac{|\beta|^{1 / 2}}{\pi} \int_{-\infty}^{+\infty} \frac{d \mu}{\mu^{2}+|\beta|} \ln \left(\frac{e^{\mu^{2}+|\beta|}-1}{e^{\mu^{2}+|\beta|}+1}\right),
$$

and we have used (87) for $c(\beta, \kappa)$. Similarly, for positive chemical potential we have

$$
\left\langle\Psi^{\dagger}\left(x_{1}\right) \Psi\left(x_{2}\right)\right\rangle_{T} \simeq e^{-x_{12} \frac{\sqrt{T}}{2} C(h / T)}, \quad h>0, \quad x_{12} \equiv\left(x_{1}-x_{2}\right) \rightarrow \infty .
$$

Both asymptotics (94) and (95) agree with the result for impenetrable bosons obtained in [3, 5, 41]. In the case of positive chemical potential we made this comparison for the exponential terms only. When $\kappa=0$ and the chemical potential $\beta$ is positive, the function $g_{\alpha}(\lambda)$ (54) possesses an index, which means that we cannot transform the RHP as in Section IVB Also, as can be seen from Appendix E, $d C(\beta, \kappa) / d \beta$ which appears in (87) becomes divergent for $\kappa=0$ and $\beta \rightarrow 0$. This means that the rigorous calculation of the pre-exponential factor for positive chemical potential and $\kappa \rightarrow 0$ would require a more sophisticated approach which we did not attempt in this work.

\section{B. The fermionic limit}

In the model (8) with $c \rightarrow \infty$ we use to describe the impenetrable anyons, in the limit of fermionic statistics parameter $\kappa \rightarrow 1$, the anyonic field-field correlator should coincide with that of free fermions. To see that Eq. (88) does indeed reduce to the free-fermionic expression, we note that Eqs. (10) - (4) show that for $\kappa=1$,

$$
C(\beta, \kappa)=0, \quad c(\beta, \kappa)=0 .
$$

This means that, in this case, $\alpha(\lambda)=1$, and the leading terms in the correlator (88) take the form:

$$
\left\langle\Psi^{\dagger}\left(x_{1}\right) \Psi\left(x_{2}\right)\right\rangle_{T}=\frac{-i \sqrt{T}}{2} \sum_{k=-1,0} \frac{e^{i x_{12} \sqrt{T} \lambda_{k}^{+}}}{\lambda_{k}^{+}},
$$

Taking into account that for $\kappa=1$, as follows from Eqs. (62) and (63),$\lambda_{0}^{+}=-\left(\lambda_{-1}^{+}\right)^{*}=(\beta+i \pi)^{1 / 2}$, one can see that Eq. (96) coincides exactly with the asymptotics of the field correlator for free fermions, and reduces to

$$
\left\langle\Psi^{\dagger}\left(x_{1}\right) \Psi\left(x_{2}\right)\right\rangle_{T}=\frac{i T}{2} e^{-a x_{12}} \sum_{ \pm} \frac{ \pm e^{ \pm i b x_{12}}}{\sqrt{h \pm i \pi T}},
$$

where

$$
a=\left(\sqrt{h^{2}+\pi^{2} T^{2}}-h\right)^{1 / 2} / \sqrt{2}, \quad b=\left(\sqrt{h^{2}+\pi^{2} T^{2}}+h\right)^{1 / 2} / \sqrt{2} .
$$

\section{The conformal limit}

For positive chemical potential and low temperatures, $\beta \rightarrow \infty$, the system is conformal. The behavior of $C(\beta, \kappa)$ in this limit is studied in Appendix E. while the zeros (62) and (63) reduce to

$$
\lambda_{0}^{+}=\sqrt{\beta}+i \frac{\pi \kappa}{2 \sqrt{\beta}}, \quad \lambda_{-1}^{+}=-\sqrt{\beta}+i \frac{\pi|\kappa-2|}{2 \sqrt{\beta}} .
$$


To make the connection with the conformal field-theory results obtained in [10, 18], we use the original variables $x=\left(x_{1}-x_{2}\right) \sqrt{T} / 2, \beta=h / T$ and the fact that in the notations used in this work, the Fermi momentum and velocity are, respectively, $k_{F}=\sqrt{h}$ and $v_{F}=2 \sqrt{h}$. Then, using Eqs. (88) and (99), we obtain the following result for the asymptotics for the field-field correlator at low temperatures. The accuracy of our calculation of the asymptotics (88), indicated by the last term in this equation, and leading to the conditions (72) and (73), implies that for $0<\kappa<2 / 3$ we can keep only one term in the asymptotic expansion of the correlator:

$$
\left\langle\Psi^{\dagger}\left(x_{1}\right) \Psi\left(x_{2}\right)\right\rangle_{T} \simeq c_{0} e^{-x_{12} \frac{\pi T}{v_{F}}\left(\frac{\kappa^{2}}{2}+\frac{1}{2}\right)} e^{i x_{12} k_{F} \kappa} .
$$

For $2 / 3<\kappa<1$, however, the two terms in the expansion are legitimate, giving

$$
\left\langle\Psi^{\dagger}\left(x_{1}\right) \Psi\left(x_{2}\right)\right\rangle_{T} \simeq c_{0} e^{-x_{12} \frac{\pi T}{v_{F}}\left(\frac{\kappa^{2}}{2}+\frac{1}{2}\right)} e^{i x_{12} k_{F} \kappa}+c_{-1} e^{-x_{12} \frac{\pi T}{v_{F}}\left[2\left(\frac{\kappa}{2}-1\right)^{2}+\frac{1}{2}\right]} e^{i x_{12} k_{F}(\kappa-2)} .
$$

The conformal result obtained in $([10,18])$ is

$$
\left\langle\Psi^{\dagger}\left(x_{1}\right) \Psi\left(x_{2}\right)\right\rangle_{T} \simeq \sum_{Q=\left\{N^{ \pm}, d\right\}} B(Q) e^{-x_{12 \frac{\pi T}{v_{F}}}\left[2 N^{+}+2 N^{-}+\frac{1}{2}+2\left(d+\frac{\kappa}{2}\right)^{2}\right]} e^{i x_{12} k_{F}(2 d+\kappa)} .
$$

One can see that the leading terms in the sum (102), which correspond to $Q=0,0,0$ and $Q=0,0,-1$, are identical (modulo some constants) to (100) and (101). The presence of the second term in Eq. (101) (and the term with $Q=0,0,-1$ in the conformal expansion) explains why close to the fermionic point the correlation function exhibits beatings, see also Eq. (97). At $T=0$, this feature of the correlation function was noticed and explained by Calabrese and Mintchev [18].

\section{CONCLUSIONS}

We have computed rigorously the large-distance asymptotic behavior of the field-field correlation functions of impenetrable anyons at finite temperature. In the process, we have also obtained a system of differential equations which characterize the correlators at any distance. Our result agrees with the predictions of the conformal field theory at low temperatures, and describes a transition in the behavior of the correlators between the impenetrable bosons realized for the statistical parameter $\kappa=0$, and free fermions at $\kappa=1$. Variation of the asymptotic behavior of the field-field correlation function with the statistics parameter $\kappa$ illustrates the role of the exchange statistics in one dimension. This variation contradicts the intuitive notion that exchange statistics is irrelevant in 1D systems of impenetrable particles, because they can not be exchanged in 1D geometry.

The next important step in the analysis of the $1 \mathrm{D}$ anyons would be to extend the results of this work to the description of the time dependence of the field-field correlators. The resulting time-, space-, and temperature-dependent correlation functions could be calculated along the lines of Ref. [31], which treated the case of impenetrable bosons. We will attempt to do this in a future publication.

\section{APPENDIX A: SHORT-DISTANCE ASYMPTOTICS}

In this appendix, we obtain the short-distance asymptotics of the potentials $B_{+-}$and $B_{++}$and as a byproduct, compute the short-distance asymptotics of the field correlator. First, we need to express the potentials in the form more amenable for short-distance computations. We start with $B_{+-}$. Multiplying both sides of the integral equation (17) which defines $f_{+}(\lambda)$ by $\gamma e_{-}(\lambda)$ one gets

$$
\begin{aligned}
\gamma f_{+}(\lambda) \sqrt{\vartheta(\lambda)} e^{-i \lambda x} & =\gamma \vartheta(\lambda)+\gamma^{2} \sqrt{\vartheta(\lambda)} e^{-i \lambda x} \int_{-\infty}^{+\infty} \sqrt{\vartheta(\lambda)} \frac{\sin x(\lambda-\mu)}{(\lambda-\mu)} \sqrt{\vartheta(\mu)} f_{+}(\mu) d \mu, \\
& =\gamma \vartheta(\lambda)+\gamma \vartheta(\lambda) \int_{-\infty}^{+\infty} \frac{1-e^{-2 i(\lambda-\mu) x}}{2 i(\lambda-\mu)} \gamma f_{+}(\mu) \sqrt{\vartheta(\mu)} e^{-i \mu x} d \mu .
\end{aligned}
$$

This equation shows that $B_{+-}$can be written as

$$
B_{+-}(x, \beta, \gamma)=\int_{-\infty}^{+\infty} s(\lambda) d \lambda
$$


where the function $s(\lambda)$ solves the following integral equation

$$
s(\lambda)-\gamma \vartheta(\lambda) \int_{-\infty}^{+\infty} \frac{1-e^{-2 i(\lambda-\mu) x}}{2 i(\lambda-\mu)} s(\mu) d \mu=\gamma \vartheta(\lambda) .
$$

In a similar fashion, we get

$$
B_{++}(x, \beta, \gamma)=\int_{-\infty}^{+\infty} e^{2 i \lambda x} s(\lambda) d \lambda
$$

where $s(\lambda)$ is the solution of the same integral equation $(\mathrm{A2}$.

For small $x$, the solution of (A2) can be expanded as

$$
s(\lambda) \equiv s(\lambda, \beta, \gamma)=\sum_{k=0}^{\infty} s_{k}(\lambda, \beta, \gamma) x^{k}
$$

where $s_{k}$ are defined by the following recursion relations

$$
s_{0}(\lambda)=\gamma \vartheta(\lambda), \quad s_{m}(\lambda)=s_{0}(\lambda) \sum_{k=0}^{m-1} \frac{(2 i)^{m-k-1}}{(m-k) !} \int_{-\infty}^{+\infty}(\mu-\lambda)^{m-k-1} s_{k}(\mu) d \mu, \quad m \geq 1 .
$$

Defining

$$
\beta_{l}(\beta, \gamma)=\gamma \int_{-\infty}^{+\infty} \lambda^{l} \vartheta(\lambda) d \lambda
$$

i.e., $\beta_{l} \equiv 0$ for odd $l$, and using (A4) in (A1) and (A3), we obtain the short-distance asymptotics for the potentials

$$
\begin{aligned}
& B_{++}(x, \beta, \gamma)=\beta_{0}+\beta_{0}^{2} x+\left(\beta_{0}^{3}-2 \beta_{2}\right) x^{2}+\left(\beta_{0}^{4}-\frac{4}{3} \beta_{0} \beta_{2}\right) x^{3}+O\left(x^{4}\right) \\
& B_{+-}(x, \beta, \gamma)=\beta_{0}+\beta_{0}^{2} x+\beta_{0}^{3} x^{2}+\left(\beta_{0}^{4}-\frac{4}{3} \beta_{0} \beta_{2}\right) x^{3}+O\left(x^{4}\right) .
\end{aligned}
$$

These relations give us the short-distance asymptotics of the field correlator. Combining them with Eqs. (33) and (37)

$$
g(x, \beta, \gamma)=\left.B_{++}(x, \beta, \gamma) e^{\sigma(x, \beta, \gamma)}\right|_{\gamma=\left(1+e^{i \pi \kappa}\right) / \pi}, \quad \sigma(x, \beta, \gamma)=-\int_{0}^{x} B_{+-}(y, \beta, \gamma) d y
$$

we find first

$$
\sigma(x, \beta, \gamma)=-\beta_{0} x-\frac{1}{2} \beta_{0}^{2} x^{2}-\frac{1}{3} \beta_{0}^{3} x^{3}+O\left(x^{4}\right)
$$

and then

$$
g(x, \beta, \gamma)=\beta_{0}\left(1-2 \frac{\beta_{2}}{\beta_{0}} x^{2}+\frac{2}{3} \beta_{2} x^{3}\right)+O\left(x^{4}\right)
$$

In the original variables $x=\left(x_{1}-x_{2}\right) \sqrt{T} / 2>0, \beta=h / T, \lambda \rightarrow \lambda / \sqrt{T}$, this result gives for the correlator (13)

$$
\left\langle\Psi^{\dagger}\left(x_{1}\right) \Psi\left(x_{2}\right)\right\rangle_{T}=D\left(1-\frac{E}{2 D}\left(x_{1}-x_{2}\right)^{2}+\gamma \frac{\pi E}{6}\left(x_{1}-x_{2}\right)^{3}\right)+O\left(\left(x_{1}-x_{2}\right)^{4}\right), \quad \gamma=\left(1+e^{i \pi \kappa}\right) / \pi
$$

where

$$
D=\frac{1}{2 \pi} \int_{-\infty}^{+\infty} \frac{d \lambda}{1+e^{\left(\lambda^{2}-h\right) / T}}, \quad E=\frac{1}{2 \pi} \int_{-\infty}^{+\infty} \frac{\lambda^{2} d \lambda}{1+e^{\left(\lambda^{2}-h\right) / T}},
$$

are the particle and the kinetic energy density, respectively. 


\section{APPENDIX B: LOW DENSITY EXPANSIONS}

As usual, the low-density limit is reached when the chemical potential is such that $\beta=h / T \rightarrow-\infty$. In our rescaled variables, the density of impenetrable anyons is given by

$$
D=\frac{\sqrt{T}}{2 \pi} \int_{-\infty}^{+\infty} \frac{d \lambda}{1+e^{\lambda^{2}-\beta}}
$$

so that $D \rightarrow 0$ for $\beta \rightarrow-\infty$. In what follows, it will be convenient to use the variable

$$
\zeta \equiv-e^{\beta}, \quad \zeta \rightarrow 0 \quad \text { for } \beta \rightarrow-\infty
$$

In order to obtain the low-density expansions for the potentials

$$
B_{++}=\sum_{k=1}^{\infty} b_{k}(x) \zeta^{k}, \quad B_{+-}=\sum_{k=1}^{\infty} c_{k}(x) \zeta^{k},
$$

we again use Eqs. (A1) and (A3), and the integral equation (A2) which has the form suitable for interation expansion in density. In terms of $\zeta$, the Fermi weight can be represented as

$$
\vartheta(\lambda)=-\sum_{k=1}^{\infty} \zeta^{k} e^{-k \lambda^{2}}
$$

Expanding also $s(\lambda)$ :

$$
s(\lambda)=\sum_{k=1}^{\infty} \zeta^{k} s_{k}(\lambda, x)
$$

we obtain from Eq. (A2) the following recursion relations for the "coefficients" $s_{k}$ :

$$
\begin{aligned}
s_{1}(\lambda) & =-\gamma e^{-\lambda^{2}}, \\
s_{k}(\lambda, x) & =e^{-\lambda^{2}} s_{k-1}(\lambda, x)-\gamma e^{-\lambda^{2}} \int_{-\infty}^{+\infty} \frac{1-e^{-2 i(\lambda-\mu) x}}{2 i(\lambda-\mu)} s_{k-1}(\mu, x) d \mu, \quad k \geq 2 .
\end{aligned}
$$

The first terms of the expansions of the potentials obtained from these recursion relations and Eqs. A1 and (A33) are:

$$
\begin{aligned}
& B_{+-}(x, \zeta, \kappa)=-\gamma \sqrt{\pi} \zeta+\left(-\gamma \sqrt{\frac{\pi}{2}}+\gamma^{2} \pi \int_{0}^{x} e^{-x_{1}^{2}} d x_{1}\right) \zeta^{2}+O\left(\zeta^{3}\right) \\
& B_{++}(x, \zeta, \kappa)=-\gamma \sqrt{\pi} e^{-x^{2}} \zeta+\left(-\gamma \sqrt{\frac{\pi}{2}} e^{-x^{2}}+\gamma^{2} \pi e^{-x^{2}} \int_{0}^{x} e^{-2 x_{1}^{2}+2 x_{1} x} d x_{1}\right) \zeta^{2}+O\left(\zeta^{3}\right)
\end{aligned}
$$

Similarly to the short-distance expansions, Eq. (B1) gives

$$
\sigma(x, \beta, \gamma)=-\gamma \sqrt{\pi} x e^{\beta}+O\left(e^{2 \beta}\right),
$$

and the correlator in the rescaled variables:

$$
\frac{\sqrt{T}}{2 \pi \gamma} g(x, \beta, \gamma)=\frac{\sqrt{T}}{2 \pi^{1 / 2}} e^{-x^{2}} e^{\beta}+O\left(e^{2 \beta}\right) .
$$

In the original variables, this result for the correlator is:

$$
\left\langle\Psi^{\dagger}\left(x_{1}\right) \Psi\left(x_{2}\right)\right\rangle_{T}=D e^{-T\left(x_{1}-x_{2}\right)^{2} / 4}
$$

It is valid as long as we can neglect the $O\left(e^{2 \beta}\right)$ terms, i.e. for $T\left(x_{1}-x_{2}\right)^{2} \ll|h| / T$. 


\section{APPENDIX C: SOLVABILITY OF THE MATRIX RIEMANN-HILBERT PROBLEM}

As we have shown in Section IVA the matrix RH problem (42) is equivalent to the system of nonsingular integral equations for functions $f_{ \pm}(\lambda)$ :

$$
f_{ \pm}(\lambda)-\gamma \int_{-\infty}^{+\infty} K_{T}(\lambda, \mu) f_{ \pm}(\mu) d \mu=e_{ \pm}(\lambda)
$$

with the kernel (15). This means that the RH problem has a unique solution whenever this system of integral equations of Fredholm type has a unique solution. To analyze these equations, we fix $\beta$ and $\kappa$, leaving the kernel $K_{T}(\lambda, \mu)$ a function of coordinate $x$. Let $D$ be an open connected subset of the complex plane and $\mathcal{L}(\mathcal{H})$ - the space of operators acting on a separable Hilbert space $\mathcal{H}$. Consider the function

$$
f(x): D \rightarrow \mathcal{L}(\mathcal{H}),
$$

which for each $x$ in $D$ gives the integral operator with kernel $K_{T}(\lambda, \mu)$. Then for each $x$ in the finite strip

$$
0<a<\Re x<b, \Im x<\epsilon,
$$

$f(x)$ is analytic operator-valued function. The kernel $K_{T}(\lambda, \mu)$ also satisfies the estimate

$$
\int_{-\infty}^{+\infty} \int_{-\infty}^{+\infty}\left|K_{T}(\lambda, \mu)\right|^{2} d \lambda d \mu<C b^{2}
$$

where $C$ is a constant, which means that $f(x)$ is compact for each $x \in D$ (see Thm. VI. 23 of [39]). Under these conditions, we can apply the analytic Fredholm theorem.

Theorem C.1 (Thm VI. 14 of [39]). Let $D$ be an open connected subset of $\mathbb{C}$. Let $f: D \rightarrow \mathcal{L}(\mathcal{H})$ be an analytic operator-valued function such that for each $z \in D, f(z)$ is compact. Then, either

a) $(I-f(z))^{-1}$ exists for no $z \in D$; or

b) $(I-f(z))^{-1}$ exists for all $z \in D \backslash S$, where $S$ is a discrete subset of $D$ (i.e. a set which has no limit points in $D)$. In this case $(I-f(z))^{-1}$ is meromorphic in $D$, analytic in $D \backslash S$, the residues at the poles are finite rank operators, and if $z \in S$, then equation $f(z) \psi=\psi$ has a nonzero solution in $\mathcal{H}$.

As a consequence of this theorem, we have to prove that for at least one point in the strip $D$ the integral equations have a unique solution. But this is definitely true for small $x$, where the Liouville-Neumann series is convergent. Thus, we have shown that the matrix RH problem has a unique solution except for a countable set of values of $x_{n}$, which we will denote by $X=\left\{x_{n}\right\}$.

\section{APPENDIX D: SCALAR RIEMANN-HILBERT PROBLEM}

This Appendix provides the basic information on the scalar Riemann-Hilbert problem used in Sec. IVB, The general problem of this type for the semi-plane is formulated as follows [40]. Consider two functions $g(\lambda)$ and $r(\lambda)$ defined on the real axis, with $g(\lambda)$ nonvanishing. Both are assumed to satisfy the Hölder condition: $\left|g\left(\lambda_{1}\right)-g\left(\lambda_{2}\right)\right|<C\left|\lambda_{1}-\lambda_{2}\right|^{k}$, and similarly for $r(\lambda)$, with some power $k$ : $0<k \leq 1$. One needs to find the function $\alpha(\lambda)$, or $\tilde{\alpha}(\lambda)$, which is analytic separately in the upper and lower half-plane, with the boundary values on the real axis satisfying the conditions:

$$
\alpha_{-}(\lambda)=\alpha_{+}(\lambda) g(\lambda), \quad \lambda \in \mathbb{R} \text { homogeneous problem, }
$$

or

$$
\tilde{\alpha}_{-}(\lambda)=\tilde{\alpha}_{+}(\lambda) g(\lambda)+r(\lambda), \quad \lambda \in \mathbb{R} \quad \text { inhomogeneous problem. }
$$

For the purposes of this work, we will assume also the normalization condition $\alpha(\infty)=\tilde{\alpha}(\infty)=1$. The considerations presented below can be extended to the more general case of a simply-connected closed contour in the complex plane - see [40]. 


\section{The homogeneous problem}

We need to distinguish three cases depending on the index $\chi(g)=(1 / 2 \pi) \operatorname{Var}_{[-\infty,+\infty]}$ arg $g(\lambda)$ of the function $g(\lambda)$. If $\chi=0$, the $\mathrm{RH}$ problem with the normalization condition is uniquely solvable. If the index is positive, $\chi>0$, the problem has $\chi+1$ linearly independent solutions, whereas the problem has no solution for $\chi<0$. For $\chi=0$, which is the situation most important for the present discussion, the solution of the RH problem (D1) is given by

$$
\alpha(\lambda)=\exp \left\{-\frac{1}{2 \pi i} \int_{-\infty}^{+\infty} \frac{\ln g(\mu)}{\mu-\lambda} d \mu\right\}, \quad \lambda \in \mathbb{C} / \mathbb{R} .
$$

As in the matrix case, it is straightforward to show that the scalar RH problem (D1) is equivalent to the singular integral equation

$$
\alpha_{+}(\lambda)=1+\frac{1}{2 \pi i} \int_{-\infty}^{+\infty} \frac{\alpha_{+}(\mu)(1-g(\mu))}{\mu-\lambda-i 0} d \mu, \quad \lambda \in \mathbb{R} .
$$

\section{The inhomogeneous problem}

Similarly to the homogeneous problem, the solution of the inhomogeneous RH problem (D2) with the normalization condition is unique for $\chi=0$, which is the situation of interest for the present discussion. The solution can be obtained from the solution of the homogeneous problem with the same $g(\lambda)$. If $\alpha(\lambda)$ solves $(\mathrm{D} 1)$, then $g(\lambda)=\alpha_{-}(\lambda) / \alpha_{+}(\lambda)$ for $\lambda \in \mathbb{R}$, and (D2) can be written as

$$
\frac{\tilde{\alpha}_{+}(\lambda)}{\alpha_{+}(\lambda)}-\frac{\tilde{\alpha}_{-}(\lambda)}{\alpha_{-}(\lambda)}=-\frac{r(\lambda)}{\alpha_{-}(\lambda)}
$$

The functions $\tilde{\alpha}_{+}(\lambda) / \alpha_{+}(\lambda)$ and $\tilde{\alpha}_{-}(\lambda) / \alpha_{-}(\lambda)$ are the boundary values of the function $\tilde{\alpha}(\lambda) / \alpha(\lambda)$ which is analytic in the complex plane minus the real axis and approaches 1 at infinity due to imposed normalization conditions $\tilde{\alpha}(\infty)=\alpha(\infty)=1$. Using the properties of the Cauchy integral, one obtains from this:

$$
\frac{\tilde{\alpha}(\lambda)}{\alpha(\lambda)}=1-\frac{1}{2 \pi i} \int_{-\infty}^{+\infty} \frac{r(\mu)}{\alpha_{-}(\mu)(\mu-\lambda)} d \mu, \quad \lambda \in \mathbb{C} / \mathbb{R} .
$$

Thus, solution of the inhomogeneous scalar RH problem (D2) is given by

$$
\tilde{\alpha}(\lambda)=\alpha(\lambda)\left(1-\frac{1}{2 \pi i} \int_{-\infty}^{+\infty} \frac{r(\mu)}{\alpha_{-}(\mu)(\mu-\lambda)} d \mu\right), \quad \lambda \in \mathbb{C} / \mathbb{R} .
$$

where $\alpha(\lambda)$ is the solution of the homogeneous problem (D1). The singular integral equation equivalent to the inhomogeneous $\mathrm{RH}$ problem is

$$
\tilde{\alpha}_{+}(\lambda)=1+\frac{1}{2 \pi i} \int_{-\infty}^{+\infty} \frac{\tilde{\alpha}_{+}(\mu)(1-g(\mu))}{\mu-\lambda-i 0} d \mu-\frac{1}{2 \pi i} \int_{-\infty}^{+\infty} \frac{r(\mu)}{\mu-\lambda-i 0} d \mu, \quad \lambda \in \mathbb{R} .
$$

\section{APPENDIX E: ANALYSIS OF $C(\beta, \kappa)$}

In this appendix, we study the behavior of the function $C(\beta, \kappa)$, which enters the asymptotics (88) of the field correlator, and is defined by Eq. (3) with condition (2), for large and small $\beta$. We start with $\beta<0$. In this case, the expansion of the logarithms in Eq. (3) according to $\ln (1-z)=-\sum_{n=1}^{\infty} z^{n} / n$ for $|z|<1$, gives:

$$
\begin{aligned}
C(\beta, \kappa) & =\frac{1}{\pi} \int_{-\infty}^{+\infty} \sum_{n=1}^{\infty}\left(\frac{(-1)^{n+1} e^{-n\left(\lambda^{2}+|\beta|\right)}}{n}+\frac{e^{i n \pi \kappa} e^{-n\left(\lambda^{2}+|\beta|\right)}}{n}\right) d \lambda \\
& =\frac{1}{\sqrt{\pi}} \sum_{n=1}^{\infty}\left(\frac{(-1)^{n+1} e^{-n|\beta|}}{n^{3 / 2}}+\frac{e^{i n \pi \kappa} e^{-n|\beta|}}{n^{3 / 2}}\right) .
\end{aligned}
$$


Therefore, the leading terms for large and small $|\beta|$ are, respectively,

$$
C(\beta, \kappa)=\frac{e^{-|\beta|}}{\sqrt{\pi}}(1+\cos \pi \kappa)+i \frac{e^{-|\beta|}}{\sqrt{\pi}} \sin \pi \kappa, \quad \beta \rightarrow-\infty,
$$

and

$$
C(\beta, \kappa)=\frac{1}{\sqrt{\pi}} \sum_{n=1}^{\infty}\left(\frac{(-1)^{n+1}+\cos n \pi \kappa}{n^{3 / 2}}\right)+i \frac{1}{\sqrt{\pi}} \sum_{n=1}^{\infty} \frac{\sin n \pi \kappa}{n^{3 / 2}}, \quad \beta \rightarrow 0
$$

When $\beta$ is small, its sign is irrelevant and, as we see explicitly below, the last equation holds both for negative and positive $\beta$.

For $\beta>0$, we can transform the logarithms in Eq. (3) so that the same expansion is applicable, and get

$$
\begin{aligned}
C(\beta, \kappa)=\frac{2}{\pi} & \int_{0}^{\sqrt{\beta}}\left[-i \pi(\kappa-1)+\sum_{n=1}^{\infty}\left(\frac{(-1)^{n+1} e^{n\left(\lambda^{2}-\beta\right)}}{n}+\frac{e^{-i n \pi \kappa} e^{n\left(\lambda^{2}-\beta\right)}}{n}\right)\right] d \lambda \\
& +\frac{2}{\pi} \int_{\sqrt{\beta}}^{\infty} \sum_{n=1}^{\infty}\left(\frac{(-1)^{n+1} e^{-n\left(\lambda^{2}-\beta\right)}}{n}+\frac{e^{i n \pi \kappa} e^{-n\left(\lambda^{2}-\beta\right)}}{n}\right) d \lambda .
\end{aligned}
$$

When $\beta$ is large, the formulae

$$
e^{-\beta n} \int_{0}^{\sqrt{\beta}} e^{\lambda^{2} n} d \lambda=\frac{1}{2 n \sqrt{\beta}}+O\left(\frac{1}{\beta^{3 / 2}}\right), \quad e^{\beta n} \int_{\sqrt{\beta}}^{\infty} e^{-\lambda^{2} n} d \lambda=\frac{1}{2 n \sqrt{\beta}}+O\left(\frac{1}{\beta^{3 / 2}}\right)
$$

simplify this expression into

$$
C(\beta, \kappa)=\frac{2}{\pi \sqrt{\beta}} \sum_{n=1}^{\infty}\left(\frac{(-1)^{n+1}}{n^{2}}+\frac{\cos n \pi \kappa}{n^{2}}\right)-2 i \sqrt{\beta}(\kappa-1)+O\left(\frac{1}{\beta^{3 / 2}}\right) .
$$

This expression can be transformed finally using the formulae $(0.234)$ and $(1.443)$ of $[42], \sum_{k=1}^{\infty}(-1)^{n+1} / n^{2}=\pi^{2} / 12$, and $\sum_{k=1}^{\infty} \cos n \pi \kappa / n^{2}=\pi^{2} B_{2}(\kappa / 2)$, where $B_{2}(x)=x^{2}-x+1 / 6$ is the second Bernoulli polynomial. This gives

$$
C(\beta, \kappa)=\frac{\pi}{\sqrt{\beta}}\left(\frac{\kappa^{2}}{2}-\kappa+\frac{1}{2}\right)-2 i \sqrt{\beta}(\kappa-1), \quad(\beta \rightarrow \infty) .
$$

Equation (E3) also shows that for $\beta \rightarrow+0, C(\beta, \kappa)$ is given by the same Eq. (E2) as for $\beta \rightarrow-0$.

[1] O.I. Pâţu, V.E. Korepin and D.V. Averin: J. Phys. A 41 (2008) 255205; arXiv:0803.0750.

[2] O.I. Pâţu, V.E. Korepin and D.V. Averin: J. Phys. A 41 (2008) 145006; arXiv:0801.4397.

[3] V.E. Korepin, N.M. Bogoliubov, and A.G. Izergin, Quantum Inverse Scattering Method and Correlation Functions, (Cambridge Univ. Press, 1993).

[4] A.R. Its, A.G. Izergin, and V.E. Korepin and N.A. Slavnov: Int. J. Mod. Phys. B4 (1990) 1003.

[5] A.R. Its, A.G. Izergin, and V.E. Korepin: Physica D 53 (1991) 187.

[6] S.J.B. Rabello, Phys. Rev. Lett. 76 (1996) 4007.

[7] U. Aglietti, L. Griguolo, R. Jackiw, S.Y. Pi, and D. Seminara, Phys. Rev. Lett. 77 (1996) 4406.

[8] A. Kundu: Phys. Rev. Lett. 83 (1999) 1275; hep-th/9811247.

[9] D.V. Averin and J.A. Nesteroff: Phys. Rev. Lett. 99 (2007); arXiv:0704.0439.

[10] O.I. Pâțu, V.E. Korepin and D.V. Averin: J. Phys. A 40 (2007), 14963; arXiv:0707.4520.

[11] M.T. Batchelor, X.-W. Guan, and J.-S. He: J. Stat. Mech. (2007) P03007; cond-mat/0611450.

[12] J.-X. Zhu and Z.D. Wang, Phys. Rev. A 53 (1996) 600.

[13] M.T. Batchelor, X.-W. Guan, and N. Oelkers: Phys. Rev. Lett. 96 (2006) 210402; cond-mat/0603643.

[14] M.D. Girardeau, Phys. Rev. Lett. 97 (2006) 100402.

[15] R. Santachiara, F. Stauffer and D.C. Cabra: J. Stat. Mech. (2007) L05003; cond-mat/0610402.

[16] A. del Campo: Phys. Rev. A 78 (2008) 045602; arXiv:0805.3786].

[17] S. Ouvry and A.P. Polychronakos: arXiv:0812.0741.

[18] P. Calabrese and M. Mintchev: Phys. Rev. B 75 (2007) 233104; cond-mat/0703117. 
[19] Y. Hao, Y. Zhang and S. Chen: Phys. Rev. A 78 (2008) 023631; arXiv:0805.1988.

[20] L. Amico, A. Osterloh and U. Eckern: Phys. Rev. B 58 (1998), 1703R; cond-mat/9803074.

[21] A. Osterloh, L. Amico and U. Eckern: J. Phys. A 33 (2000) L87 cond-mat/9812317; L487 cond-mat/0007081.

[22] M.T. Batchelor, A. Foerster, X.-W. Guan, J. Links, H.-Q. Zhou: J. Phys. A 41 (2008) 465201; arXiv:0807.3197.

[23] Y. Hao, Y. Zhang and S. Chen: arXiv:0901.1224.

[24] M. T. Batchelor, X.-W. Guan and A. Kundu: J. Phys. A 41 (2008) 352002; arXiv:0805.1770].

[25] P. Calabrese and R. Santachiara: J. Stat. Mech. P03002 (2009); arXiv:0811.2991.

[26] V. V Cheianov and M. B. Zvonarev: J. Phys. A 37 (2004) 2261-2297 cond-mat/0310499.

[27] V. V Cheianov and M. B. Zvonarev: Phys. Rev. Lett. 92 (2004) 176401 cond-mat/0308470.

[28] O.I. Pâţu, V.E. Korepin and D.V. Averin: EPL 86 (2009) 40001 arXiv:0811.2419.

[29] Wu T.T., B.M. McCoy, C.A. Tracy and E. Barouch: Phys. Rev B 13 (1976) 316.

[30] M. Jimbo, T. Miwa, Y. Môri, and M. Sato: Phys. D 2 (1981) 80.

[31] A.R. Its, A.G. Izergin, and V.E. Korepin and G.G. Varzugin: Physica 54 D (1992) 351.

[32] J. Harnad, A.R. Its: Comm. Math. Phys. 226 (2002) 497.

[33] A.R. Its, A.G. Izergin, and V.E. Korepin: Comm. Math. Phys. 129 (1990) 205.

[34] R. Santachiara and P. Calabrese: J. Stat. Mech. P06005 (2008) arXiv:0802.1913.

[35] P.J. Forrester, N.E. Frankel, T.M. Garoni and N.S. Witte: Comm. Math. Phys. 238 (2003) 257 math-ph/0207005.

[36] N. Kitanine, K. K. Kozlowski, J. M. Maillet, N. A. Slavnov, V. Terras: arXiv:0805.4586.

[37] P. Deift and X. Zhou: Ann. of Math. (2) 137 (1993) no.2, 295.

[38] L.D. Faddeev and L.A. Takhtajan, Hamiltonian Methods in the Theory of Solitons, (Springer-Verlag Berlin Heidelberg 2007).

[39] M. Reed and B. Simon, Methods of Modern Mathematical Physics I. Functional Analysis, (Academic Press 1980).

[40] F.D. Gakhov, Boundary Value Problems, (Pergamon Press 1966).

[41] A.R. Its, A.G. Izergin, and V.E. Korepin: Comm. Math. Phys. 130 (1990) 471.

[42] I.S. Gradshteyn and I.M. Ryzhik, Table of Integrals, Series, and Products, (Academic Press, 2007). 